\title{
Co - processing of pharmaceutical cocrystals for high quality and enhanced physicochemical stability
}

Steven A. Ross ${ }^{1}$, Adam Ward ${ }^{2}$, Pat Basford ${ }^{3}$, Mark McAllister ${ }^{3}$, Dennis Douroumis ${ }^{1,4 *}$

1 Faculty of Engineering and Science, School of Science, University of Greenwich, Medway Campus, Chatham Maritime, Kent ME4 4TB, UK.

2 Department of Pharmacy, School of Applied Sciences, University of Huddersfield, Huddersfield, West Yorkshire, HD1 3DH, UK

3 Pfizer Global Research \& Development, Ramsgate Road, Sandwich, CT13 9NJ, UK

4 Medway School of Pharmacy, University of Kent, Medway Campus, Central Avenue, Chatham Maritime, Chatham, Kent ME4 4TB, UK

ABSTRACT: The physical and chemical stability of indomethacin-saccharin cocrystals was enhanced through co-processing with inert excipients at the final conveying zone during hot-melt extrusion. The synthesized cocrystals were co-processed with a crystalline hydrophilic polymer (PEG 6000), an amorphous hydrophilic polymer (hydroxypropylmethyl cellulose, HPMC) and an aluminometasilicate inorganic (Neusilin) excipient. Physiochemical characterization of the suspended cocrystals in the Neusilin and HPMC carriers revealed superior stability and the absence of any interactions between the excipients and the parent cocrystals. In contrast extruded cocrystals not suspended in any excipient or co-processed with PEG 6000 underwent disassociation under accelerated conditions. Surface dissolution analysis demonstrated that Neusilin and PEG 6000 have no effect on the cocrystal dissolution rates $(>90 \%)$ while HPMC led to in-situ gelling and hence slower rates ( 4\%). In conclusion, cocrystals with high crystallinity and improved physicochemical stability can be synthesised by co-processing with inert, non-miscible, low viscosity and thermally stable excipients.

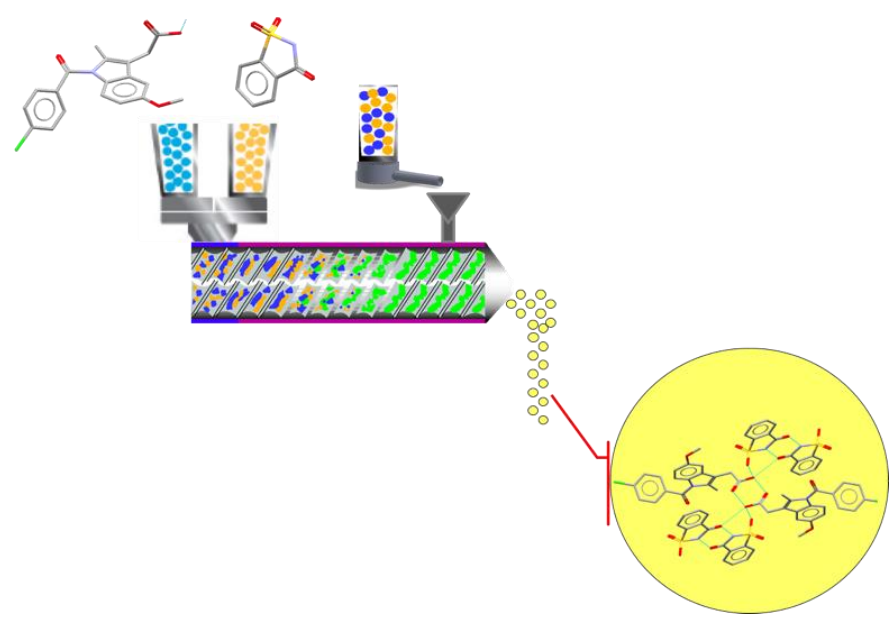

*Department of Pharmaceutical, Chemical \& Environmental Sciences, Faculty of Engineering and Science, University of Greenwich, Medway Campus, Chatham Maritime, Kent, ME4 4TB, UK. Tel.: +44(0)2083318440; Fax: +44(0)2083319805. E-mail: D.Douroumis@gre.ac.uk. 


\section{$\mathrm{Co}$ - processing of pharmaceutical cocrystals for}

\section{high quality and enhanced physicochemical stability}

Steven A. Ross ${ }^{1}$, Adam Ward $^{2}$, Pat Basford ${ }^{3}$, Mark McAllister ${ }^{3}$, Dennis Douroumis ${ }^{1}, 4^{*}$

1 Faculty of Engineering and Science, School of Science, University of Greenwich, Medway Campus, Chatham Maritime, Kent ME4 4TB, UK.

2 Department of Pharmacy, School of Applied Sciences, University of Huddersfield, Huddersfield, West Yorkshire, HD1 3DH, UK

3 Pfizer Global Research \& Development, Ramsgate Road, Sandwich, CT13 9NJ, UK

4 Medway School of Pharmacy, University of Kent, Medway Campus, Central Avenue, Chatham Maritime, Chatham, Kent ME4 4TB, UK

\footnotetext{
* Correspondence: Medway School of Science, Universities of Greenwich \& Kent, Medway Campus, Chatham Maritime, Kent ME4 4TB, UK. Tel.: +44 (0) 208331 8440; Fax: +44 (0) 208 331 9805. E-mail address: D.Douroumis@gre.ac.uk
} 


\begin{abstract}
Solid state synthesis of high-quality indomethacin-saccharin cocrystals was achieved using Hot Melt Extrusion. The physical and chemical stability of the formed cocrystals was enhanced through co - processing with inert excipients at the final kneading zone. For the purposes of the study the synthesized cocrystals were co-processed with a crystalline hydrophilic polymer (PEG 6000), an amorphous hydrophilic polymer (hydroxypropylmethyl cellulose, HPMC) and an aluminometacilicate inorganic (Neusilin) excipient. Physiochemical characterization of the suspended cocrystals in the Neusilin and HPMC carriers revealed superior stability and the absence of any interactions between the excipients and the parent cocrystals. In contrast extruded cocrystals that were not suspended in any excipient or co-processed with PEG 6000 underwent disassociation under accelerated conditions. Surface dissolution analysis demonstrated that Neusilin and PEG6000 have no effect on the cocrystal dissolution rates $(>90 \%)$ while HPMC led to in - situ gelling effect and hence in slower rates $(\sim 4 \%)$ In conclusion, cocrystals with high crystallinity and improved physicochemical stability can be synthesised by co-processing with excipients that are inert, non- miscible, have good thermal stability and a low viscosity.
\end{abstract}

Keywords: Cocrystals, Hot Melt Extrusion, co - processing, stability 


\section{Introduction}

A cocrystal is a multicomponent molecular crystal in which all components are at a stoichiometric ratio and comprise of two or more chemically different molecules. ${ }^{1,2}$ Cocrystal synthesis involves the formation of supramolecular heterosynthons of certain functional groups which are non-covalently bonded. These molecular interactions result in either $\mathrm{H}-$ bonding or the formation of van der Waals forces which make up the structure of thermodynamically stable cocrystals. ${ }^{3}$ Cocrystallization offers an expedient way to alter the physicochemical properties of APIs including dissolution rate, intrinsic solubility, melting point, hygroscopicity, compressibility, bulk density and friability. ${ }^{4}$ There has been a recent surge in industrial interest into cocrystals due in part to their potential to improve the physicochemical properties of poorly bioavailable API; advances in continuous manufacturing technologies, leading to fewer production steps and increased cost effectiveness; improving regulatory and intellectual property factors, potential for green synthesis and the innate advantages to working with known API as opposed to new drugs. $5-7$

Pharmaceutical cocrystals tend to exhibit specific physical stability issues. One of the biggest examples of these issues is that caused by hydrate formation. Due to their small size and multidirectional hydrogen bonding abilities, it is common for the API of a cocrystal to form a hydrate with water molecules. This is particularly common at higher temperatures and humidities due to moisture uptake from the atmosphere, potentially converting the drug into its hydrated form. Reversible hydrate formation indicates that neither the anhydrate nor the hydrate is physically stable across the range of common processing conditions. ${ }^{8}$ This can be observed in the case of Theophylline/citric acid cocrystals where the cocrystal was observed to convert to the cocrystal hydrate after 3 days at $75 \%$ relative humidity $(\mathrm{RH}) .{ }^{9}$ 
It has been shown that coformers which modulate both solubility and water activity, such as citric acid or saccharin, are predisposed to conversions due to the moisture uptake at high humidities and the coformers deliquescence causing dissolution into the absorbed water. ${ }^{10-12}$ The removal and/or alteration of the coformer will result in the disassociation of the parent API crystals, formation of API hydrate crystals or other cocrystal forms which can potentially lower the solubility and reduce the bioavailability of the drug. ${ }^{13}$ Other studies have noted the occurrence of cocrystal dissociation due to partial dissolution of one of the parent compounds at elevated temperatures and humidities. Eddleston et al. (2014) reported that Theophylline and Caffeine cocrystals with dicarboxylic acid coformers experienced partial dissociation at $75 \%, 85 \%$ and $95 \%$ $\mathrm{RH}$, due to partial dissolution of the coformers. ${ }^{14}$ The cocrystals dissociated due to aqueous solubility differences between the API and coformers, causing coformer dissolution and recrystallization of the bulk API. This has been noted in API-coformer pairs whose relative solubilities differ by as little as $30 \% .{ }^{15}$

An emerging approach designed to increase the chemical and physical stability of cocrystals involves co-processing the API-coformer pairs alongside inert excipients. This approach, dubbed matrix-assisted cocrystallization (MAC) in one study, sees the API-coformer pair blended with an inert third material prior to or during a hot-melt extrusion (HME) process. ${ }^{16-19}$ Using the typical MAC approach, all constituents are pre-mixed and fed into the extruder together. Cocrystallization occurs across the extruder barrel, facilitated by intimate grinding at temperatures near the melting or softening point of the added excipient. This causes the cocrystals to become imbedded in a protective matrix once the excipients solidify.

This approach was first conceived by Liu et al. (2011), who co-extruded carbamazepine/nicotinamide cocrystals with HPMC, Soluplus ${ }^{\circledR}$ (PVAc-PVCap- PEG) or PVP/VA 
polymer excipients (60:40 amounts). ${ }^{16}$ Here, cocrystals formed in-situ before melting at $160{ }^{\circ} \mathrm{C}$ and becoming completely dispersed in the polymer carrier forming an amorphous solid dispersion. However, X-ray analysis revealed that prior to melting the cocrystals were embedded in the polymer matrices. This was expanded upon by Boksa et al. (2014) who employed a similar MAC method, but this time at lower temperatures with the intent to form cocrystals as opposed to solid dispersions. ${ }^{17}$ Here, carbamazepine-nicotinamide cocrystals co-processed with Soluplus ${ }^{\circledR}$ were found to produce cocrystals embedded in a polymer matrix. Partial least square regression models and X-ray powder diffraction (XRPD) revealed the extruded cocrystals found only $0.3 \%( \pm 2.9)$ of unconverted CBZ and NIC in the extruded samples. ${ }^{17}$

A recent study Li et al. (2016) helped shed some light on the quality's excipient must possess to allow the formation high quality cocrystals. ${ }^{18}$ The added excipient must be completely inert and not miscible with the API- coformer pair, have a sufficiently lower processing temperature than the cocrystal constituents, have a low melting viscosity and must solidify quickly upon cooling. Thus, the added excipient is inert is of utmost importance, as any strong non-covalent interaction between constituents other than between the API-coformer pair has the potential to negatively affect cocrystal yield. XRPD analysis of MAC extruded Ibuprofen (IBU) - Isonicotinamide (IsoNA) cocrystals processed alongside either EPO or Soluplus ${ }^{\circledR}$, showed absence of characteristic peaks associated with the IBU-IsoNA cocrystal, indicating a reduction in yield. This is likely because the highly miscible polymers interacted with the IBU, hindering interaction with the coformer. The highly viscous Soluplus would have prevented interaction between the API and coformer in its liquid state. This is also true for the EPO and is exacerbated by the polymers tendency to form hydrogen bonds with carboxylic acids, causing competition for the IsoNA coformer, hindering cocrystallization. 
Conversely, extrusion of IBU-IsoNA cocrystals was possible when processed alongside Xylitol. This can be attributed to the tendency for polyols to be inert during extrusion, and xylitol's low viscosity combined with is decreased miscibility allowing for increased molecular mobility and the rapid solidification. ${ }^{17-20}$ However, a remaining barrier to the success of this method was the presence of amorphous IBU existing within the inert xylitol matrix, a trend which has been observed in previous studies. ${ }^{16-18}$ A possible explanation for this is that the addition of any matrix, in high concentrations will hinder the interactions of the API-coformer pair at a molecular level. This is due to the increased interactions made possible due to the high-shear kneading zones of the extruder causing dispersive mixing and thus encouraging interaction between the excipient and drug-coformer pair. ${ }^{21}$ It is possible that if the excipients were added in low concentrations at a later stage in the extrusion process, avoiding the kneading zones, that the excipient will act merely as a protective matrix and not interact with the cocrystals.

In this study, indomethacin-saccharin cocrystals were co-processed with three different inert excipients, which will be fed separately into the extruders conveying zones after the final extruder kneading zone. The three excipients chosen for this work are Neusilin (NEU), hydroxypropyl methylcellulose (HPMC) and Polyethylene glycol (PEG) 6000. NEU, a synthetic amorphous form of magnesium aluminometasilicate, is an inert absorbent excipient with thermally stability up to $700{ }^{\circ} \mathrm{C}$ and has been known to help stabilize moisture sensitive APIs. ${ }^{22,23}$ HPMC is an inert, amorphous, hydrophilic, polysaccharide with good thermal stability. PEG 6000 is a crystalline, hydrophilic polymer, which is water soluble. Due to the relatively low melting point (60-64C) of PEG 6000, the extrusion process will operate well above the melting point of this particular excipient. The reason for this is to observe how the excipient will affect the quality of the cocrystal when completely melted, but still beyond the kneading zones. To examine the effect of excipient 
concentration/loading on crystallinity, two batches for each excipient were processed, one with a $5 \%$ excipient loading and another with $10 \%$ excipient loading.

It is anticipated that by feeding the excipients into the extruder at a later stage, there will be insufficient mechanical energy and dispersive mixing to allow significant molecular interactions between the cocrystal and the excipient, while still allowing the formation of a protective matrix around the cocrystals. This protective matrix should enhance the long-term stability of the cocrystals and preserve crystallinity.

\section{Materials and methods}

\section{Materials}

Indomethacin ( $\gamma$-form, IND, $>98.0 \%)$ was purchased from Tokyo Chemical Industry Co., Ltd (Tokyo, Japan). Both Saccharin (SAC, >99\%) and Polyethylene glycol 6000 (PEG 6000) were purchased from Sigma-Aldrich (Gillingham, Dorset, UK). Neusilin US2 (NEU, >99\%) and hydroxypropyl methyl cellulose grade PHARMACOAT 603 (3 cP) were by kindly donated by Fuji Chemical Industries Co., Ltd. (Kamiichi, Toyama, Japan) and ShinEtsu (Tokyo, Japan) respectively, and were used as received. All solvent used for HPLC analysis were of analytical grade (Fisher Chemical, Loughborough, UK).

\section{Hot-melt extrusion Processing}

A $100 \mathrm{~g}$ physical mixture of IND and SAC (1:1 stoichiometric ratio) was prepared by accurately weighting and then homogeneously blending in a Turbula TF2 mixer (Willy A. Bachofen AG, Switzerland) at 100rpm for 10 minutes. The blend was then placed inside a single-screw volumetric feeder (Brabender Technology, Duisburg, Germany), where the feed rate was 
calibrated before being fed into a $16 \mathrm{~mm}$ twin screw extruder (Eurolab 16, Thermo Fisher, Germany). A second single screw gravimetric feeder of our own construction was placed above an entry port just after the final kneading zone, to feed excipients onto desired batches mid-process. This was not present for the unmodified cocrystal batches. This feeder was loaded with either NEU, HPMC or PEG 6000 in amounts equivalent to $5 \%$ or $10 \%$ of the cocrystal mixture amount. Travel time was calculated from the moment the blended mixture was first fed into the extruder to the moment it reached the second feeder. The time for the entire batch to pass this point was also taken. This was necessary to ensure the added excipient was equally dispersed throughout the entire cocrystal batch. The feed rate of the second feeder was calibrated in preliminary experiments to allow the amount of excipient to be evenly distributed throughout the cocrystal batch. This experiment was conducted without a die. Complete temperature profile is as follows: $50{ }^{\circ} \mathrm{C}, 70$ ${ }^{\circ} \mathrm{C}, 95{ }^{\circ} \mathrm{C}, 155^{\circ} \mathrm{C}, 165{ }^{\circ} \mathrm{C}, 165^{\circ} \mathrm{C}, 165{ }^{\circ} \mathrm{C}, 165{ }^{\circ} \mathrm{C}, 165^{\circ} \mathrm{C}$.

\section{Differential scanning Calorimetry (DSC)}

The thermal profiles for the Bulk IND and SAC, a physical mixture of the two, unmodified IND/SAC cocrystals and the excipient blended IND/SAC cocrystals were examined using a differential scanning calorimeter (Mettler Toledo 823e, Greifensee, Switzerland). The unmodified cocrystals and excipient blended cocrystals were further analysed after 4 weeks storage under accelerated conditions to check for changes in crystallinity. Each pan was analysed from 25-250 ${ }^{\circ} \mathrm{C}$ (with the exception of the bulk IND, which was heated from $25-250{ }^{\circ} \mathrm{C}$ ) at a heating rate of 10 ${ }^{\circ} \mathrm{C} / \mathrm{min}$. Nitrogen was utilized as the purge gas and was supplied at $50 \mathrm{~mL} / \mathrm{min}$. STARe excellence Software was used for the data integration and evaluation. 


\section{X-ray Powder Diffraction (XRPD)}

The crystallinity of bulk components, physical mixtures, unmodified cocrystals and excipient blended IND/SAC cocrystals were determined using a Bruker D8 Advance X-ray diffractometer (Karlsruhe, Germany) in theta-theta mode. A Cu anode powered at $40 \mathrm{kV}$ and $40 \mathrm{~mA}$, a primary $4^{\circ}$ Soller slit and a secondary $2.5^{\circ}$ Soller slit, and a $0.2 \mathrm{~mm}$ exit slit was selected for this experiment. A LynxEye Position Sensitive detector with $3^{\circ}$ opening, set at $6.5 \mathrm{~mm}$ was utilized and a primary Göbel mirror was used for the parallel beam and the removal of $\mathrm{CuK} \beta$. Sample rotation was set at $15 \mathrm{rpm}$ and data was collected between $2-55^{\circ} 2 \theta$ with a step size set at $0.02^{\circ} 2 \theta$ and the counting time set at 0.3 secs per step. EVA phase analysis software (Bruker, Germany) was used to identify peak positions and intensities of the bulk and extruded samples. Rietveld refinements were conducted using TOPAS V4.2 (Bruker, Germany), to validate the crystallinity of the pure and excipient blended IND/SAC cocrystal samples and compare to documented references taken from the Cambridge structural database (CSD). ${ }^{24}$ XRPD analysis and Rietveld refinements were again conducted on all samples after stability studies to observe changes after being held under accelerated conditions. CSD crystal structures retrieved and used for Rietveld refinements; IND ( $\gamma$-form) IND Refcode: INDMET02, ${ }^{25}$ SAC Refcode: SCCHRN02, ${ }^{26}$ and IND/SAC cocrystal Refcode: UFERED ${ }^{27}$.

\section{Scanning electron microscopy (SEM)}

The morphology of the bulk components, physical mixtures, unmodified and excipient blended IND/SAC cocrystals was observed via scanning electron microscope (EVO MA10, Ziess, Germany). Samples were placed on a thin aluminium stub glazed with Mikrostik nonconductive 
adhesive (Agar scientific, UK) and chromium coated under an argon atmosphere. The accelerating voltage of the electron beam was $11 \mathrm{kV}$.

\section{Particle size analysis}

Laser diffraction was used to measure the particle size distribution of the bulk components, unmodified and excipient blended IND/SAC cocrystals. This was achieved using a dry powder dispersion unit (Scirocco 2000) of a Mastersizer 2000 laser diffraction particle size analyser (Malvern, Worcestershire, UK). 5g of powder from each sample was placed in a vibratory tray which in turn fed the powder into the sample dispersion unit. Sampling time was set at 15 seconds and each sample was measured in triplicate.

\section{In vitro dissolution study}

Dissolution studies were carried out using a USP II apparatus (Varian 705 DS, North Carolina, US). Amounts of bulk IND, unmodified IND/SAC cocrystal and all excipient blended IND/SAC cocrystals equivalent to $25 \mathrm{mg}$ of IND were placed in a dissolution vessel filled with $900 \mathrm{~mL}$ of phosphate buffer solution ( $\mathrm{pH}$ 6.8). The temperature of the medium was maintained at $37^{\circ} \mathrm{C}$ and paddle rotation was set at $100 \mathrm{rpm}$. Samples were extracted via syringe in amounts of $2 \mathrm{~mL}$ at 15 , 30, 60 and 120 min intervals, before being passed through $200 \mu \mathrm{m}$ filter.

\section{HPLC analysis}

Analysis was carried out using a HPLC system (Agilent technologies, 1200 series, Massachusetts, USA) equipped with a quaternary pump. The mobile phase consisted of methanol/water/acetic acid with a 70/30/0.2 ratio (v/v/v). A Hichrom S50DS2-4889 $(5 \mu \mathrm{m} \times$ 
$150 \mathrm{~mm} \times 4 \mathrm{~mm}$ ) column was used, with the temperature set at $25^{\circ} \mathrm{C}$. The injection volume was set at $20 \mu \mathrm{m}$ and the flow rate was maintained at $1.5 \mathrm{~mL} / \mathrm{min}$ with a 4 minute retention time. The eluent was monitored with a UV detector set at a wavelength of $260 \mathrm{~nm}$. The calibration curve for IND was prepared with a concentration range of $10-50 \mu \mathrm{g} / \mathrm{mL}$.

\section{Surface dissolution imaging (SDI)}

The dissolution behaviour and intrinsic dissolution rate (IDR) for the unmodified and all excipient blended IND/SAC cocrystals were studied using a Sirius SDI 300 (Forest Row, UK) fitted with ActiPix UV area imaging technology. The dissolution imaging experiments were performed at $37^{\circ} \mathrm{C}$ at a single wavelength filter of $280 \mathrm{~nm}$. The dissolution occurred in $200 \mathrm{mM}$ of phosphate buffer $(\mathrm{pH} 6.5)$ at a flow rate of $0.2 \mathrm{~mL} / \mathrm{min}$. Each sample was analysed for 20 minutes in triplicate. Compacts were compressed at a constant pressure $(40 \mathrm{cN} . \mathrm{m})$, for $1 \mathrm{~min}$ using a Quickset minor torque wrench (Torqueleader, M.H.H. engineering Co. Ltd., Guildford, UK). Calibration was performed through UV imaging a set of varying concentration IND standard solutions in distilled water. The calibration curve was constructed by plotting absorbance against the concentration of each standard solution. IDR values were determined using calculated extinction coefficients for each of the compounds.

\section{Stability studies}

The unmodified IND/SAC cocrystals and excipient blended cocrystals were placed in a sealed desiccator and kept under accelerated conditions of $40 \pm 1{ }^{\circ} \mathrm{C}$ and $75 \pm 1.5 \% \mathrm{RH}$ as per ICH guidelines, for a period of 4 weeks to ascertain the stability of the cocrystals at accelerated conditions. ${ }^{28}$ To ensure the experimental conditions remained within the recommended limits, the 
desiccator was kept in a temperature-controlled oven with the humidity verified daily using a hygrometer.

\section{Results and Discussion}

\section{Structural analysis}

Indomethacin-Saccharin cocrystals were chosen as the model drug for this work due to the extensive amount of coverage this particular cocrystal pair has received, leaving little chance for unexplained occurrences. A quick screening using Mercury CSD 3.9 revealed that IND-SAC cocrystal contains a robust interaction between the carboxylic acid homodimer synthon found in the IND and the strong N-H donor group found in the SAC component (Figure 1). ${ }^{26}$ In addition to this, the formation of a second imide dimer synthon has been reported, due to the lack of steric effects in relation to the carboxylic acid dimer. These carboxylic acid groups have been reported to form vigorous supramolecular synthons throughout the cocrystal lattice, resulting in greater thermostability than indomethacin cocrystals prepared with, for example, a coformer containing an amide group such as nicotinamide. ${ }^{27}$ Further the screening revealed the cocrystal to have high H-bond propensities and easily accessible crystal geometries resulting in a strong and stable cocrystal pair, ideal for testing with this work. 

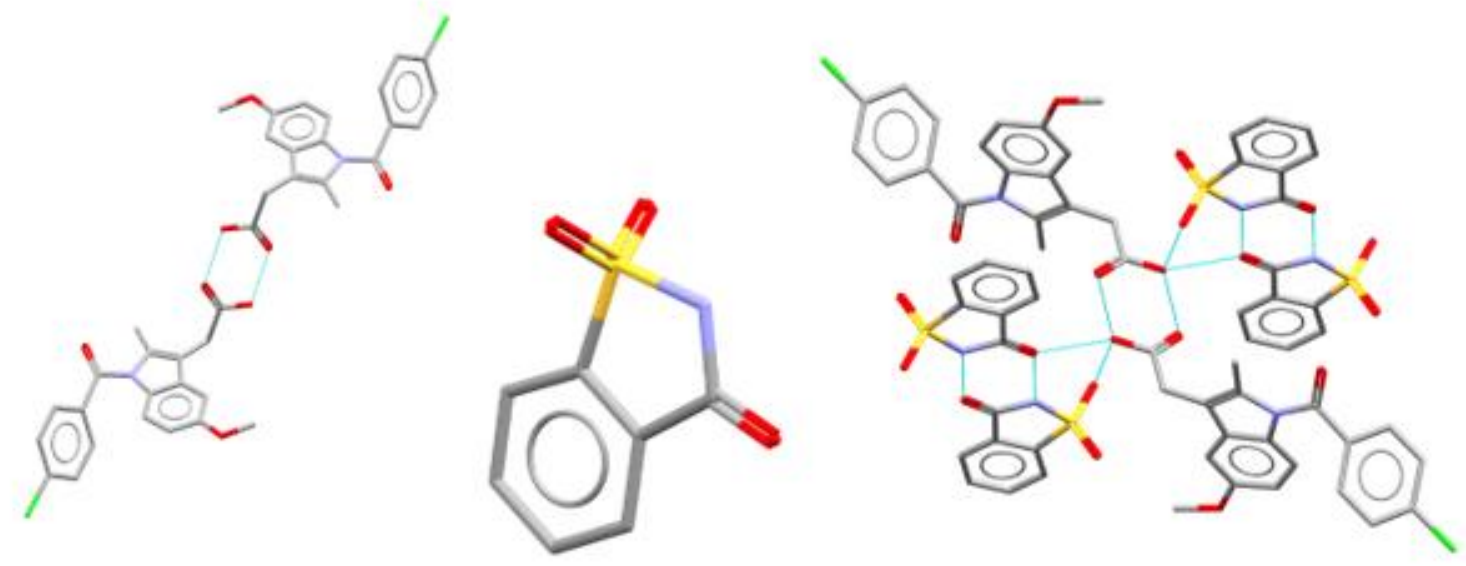

Figure 1: molecular models of A: Two molecules of Indomethacin ( $\gamma$-form), forming a robust carboxylic acid dimer synthon. B: Single Molecule of Saccharin. C: Indomethacin-Saccharin Cocrystal interconnected via a Carboxylic acid homodimer synthon and Imide dimer synthons with $\mathrm{N}-\mathrm{H} \cdots \mathrm{O}$ bonds.

\section{Hot Melt Extrusion Continuous Processing}

To accommodate the addition of the second feeder, the screw configuration had to be rearranged to ensure the excipients were fed into the device after the final kneading zone. The final kneading zone was shortened to allow for this, with three conveying elements being removed and the kneading elements pushed further down the screw in their place. The bulk cocrystal constituents were fed into the extruder at the beginning of the screw barrel through the first entry point. The excipients were fed into the barrel through a second feeder set up after the third kneading zone, through the final entry point, before the discharge zone. In total, the kneading elements were assembled in three separate "mixing zones" with 4 separate conveying zones, including one extended conveying zone towards the end of the extruder starting at the entry point for the second feeder, where the excipients were added (Images available in supporting information Figure S1, $\mathrm{S} 2$ and S3). Screw configuration was arranged with kneading zone components set at $90^{\circ}$ angles 
to allow for more aggressive, disruptive mixing. ${ }^{29}$ DSC analysis indicates that this alteration allowed complete cocrystallization to occur by the $4^{\text {th }}$ entry point, so excipients were added after the cocrystals had fully formed. Screw speed, temperature profile and feed rate were then adjusted to find the optimal processing parameters. (Table of trials available in supporting information Figure S4). Dhumal et al. (2010) presented the importance of processing parameters such as screw speed, barrel temperature profile and screw configuration on the yield and purity of extruded cocrystals, emphasizing the effect the screw configuration has in creating enough energy to encourage cocrystallization. ${ }^{30}$ By calibrating the excipients feed rate to correlate with the cocrystal feed rate it was possible to ensure all excipients were evenly distributed along the entire cocrystal batch.

The high screw temperature should prove sufficient energy to liquefy/soften the excipient while the screw rotation should allow it to intersperse around the already co-crystallized API-coformer pair. However, as the mixture will not pass through a kneading zone, there will be insufficient mechanical energy and dispersive mixing to allow significant molecular interactions between the cocrystal and the excipient. As the excipient is not interspersed throughout the crystal lattice it should instead act merely as a protective matrix for the cocrystal. It is hoped that this matrix will hinder moisture uptake and discourage hydrogen bonding at high temperatures and humidities. This will prevent the solubilisation of the coformer due to aqueous solubility differences, leading to cocrystal disassociation and protect against hydrate formation. ${ }^{14,31,32}$

\section{Thermal analysis}

The thermal properties of the bulk powders, physical mixture and unmodified IND/SAC can be seen in the supporting information (Figure S5). The bulk IND displayed a sharp melting peak at 
$160.9{ }^{\circ} \mathrm{C}$, with the SAC showing a sharp melting point at $226.9{ }^{\circ} \mathrm{C}$, in accordance with reported thermal behaviour. ${ }^{27}$ For the physical mixture, an endothermic peak can be observed at $157.1{ }^{\circ} \mathrm{C}$, immediately followed by an exothermic event at $159.2^{\circ} \mathrm{C}$. The endothermic peak can be attributed to a eutectic melting of the components, with the small exothermic event indicating the point in which cocrystallization has taken place. ${ }^{33}$ This shows that cocrystallization is likely between the two components and also reveals that spontaneous cocrystal formation had not taken place during the preparation of the physical blend. Had cocrystallization already occurred a single cocrystal melt would be shown as opposed to the cocrystal forming point observed at $\operatorname{Tmax} 159.2{ }^{\circ} \mathrm{C} .{ }^{34} \mathrm{~A}$ more intense, narrow endothermic peak can be seen at $183.2^{\circ} \mathrm{C}$, which according to Basavoju et al. (2008) is in accordance with the melting point of the IND/SAC cocrystal. ${ }^{27}$ The thermal profile of the unmodified IND/SAC cocrystal displays a single endotherm for the melting point of the cocrystal with an absence of any eutectic event or unbound/absorbed solvent or water present. This demonstrates the stability of the phase until the melting indicating complete batch cocrystallization and a high cocrystal purity. ${ }^{35}$

The thermogram for the excipient blended IND/SAC cocrystals display a near identical thermal profile to that of the unmodified IND/SAC cocrystal, with an absence of any eutectic melt or unbound solvents/water. This indicates that the addition of the excipients has had minimal effect on the cocrystal yield or purity, demonstrating that excipient blending after the kneading zones has not hindered API-coformer interaction or encouraged the formation of amorphous content.

Further DSC analysis was performed after leaving the samples under accelerated conditions for a period of 4 weeks to observe the effect of added excipients on the physical stability of IND/SAC cocrystals. The DSC thermogram for 5\% and 10\% NEU blended cocrystals alongside the unmodified IND/SAC cocrystals, before and after stability studies can be seen in Figure 2a. An 
additional endothermic peak is present at $159.98^{\circ} \mathrm{C}$ for the unmodified IND/SAC cocrystals after being held under accelerated conditions. This endothermic event can be attributed to the presence of recrystallized IND, as the peak position correlates with that of the bulk API. There is a slight melting point depression of approximately $1{ }^{\circ} \mathrm{C}$ from the usual melting point of IND, but this is a well-documented occurrence for recrystallized components in cocrystals. ${ }^{36}$ This indicates the cocrystal has undergone partial disassociation, likely as a result of dissolution of the SAC coformer at high humidities, causing the recrystallization of unbound IND. This type of cocrystal disassociation is common at humidities of $75 \% \mathrm{RH}$ when there is a large solubility difference between the API-coformer pair. ${ }^{14,37}$ It is unlikely that hydrate formation had taken place. If the cocrystal had dissociated as a result of hydrate formation desorption, evaporation or hydrate decomposition in the form of sublimation would have be observed, though the fact that a single, sharp endotherm remains in all excipient blended samples indicates no hydrate molecule is present in the cocrystal blends. The presence of bound or unbound water molecules on in the cocrystal lattice would cause other endothermic events to show in the thermal profile or a significant melting point depression, neither of which is present in the excipient blended samples, indicating the system is stable. ${ }^{38}$ In contrast, both the 5\% and $10 \%$ excipient blended samples show no such endotherm, demonstrating phase stability until the melting point of the cocrystal. This suggests that the NEU matrix has hindered any reaction between the SAC coformer and water molecules under the increased humidity conditions, thus preventing dissolution of the conformer and improving the physical stability of the cocrystal.

Equivalent data were observed for IND/SAC cocrystals blended with either HPMC or PEG 6000. As illustrated in Figure 2b, it can be observed that the HPMC blended cocrystals displayed excellent stability up until the melting point for both the $5 \%$ and $10 \%$ loaded samples after stability 
testing, indicating that the HPMC matrix has sufficiently protecting the SAC coformer from dissolving and maintaining cocrystal integrity. A small, endotherm can be seen in HPMC blended IND/SAC cocrystals samples at $242{ }^{\circ} \mathrm{C}$, after the cocrystal melting point. This is likely to be caused by the degradation of HPMC left in the pan. Like many other polysaccharides, HPMC will start to degrade rather than exhibit melting behaviour and HPMC has been known to undergo physical degradation between $225-265^{\circ} \mathrm{C} .{ }^{39}$ This is not an issue for the analysis of the thermal profiles, as it occurs after cocrystal melting. Somewhat surprisingly, the IND/SAC cocrystals blended with PEG 6000 also displays excellent thermal stability in contrast to the unmodified cocrystal sample. As the cocrystals were extruded above PEG 6000s melting point, it was though that interactions between cocrystal and excipient were possible. However, the DSC thermogram (Figure 2c) displays only one observable event, which correlates to the cocrystal melt, indicating that no further interaction between the PEG and bulk excipients has taken place. This is perhaps due to the fact that the excipients were added into the extruder in the conveying zones after complete cocrystallization had already occurred, preventing competition between the coformer and excipient to bind with the IND. That PEG 6000 quickly solidified after discharge from the extruder would have given limited time for interaction to occur, which may be another factor contributing to the excipients success. 


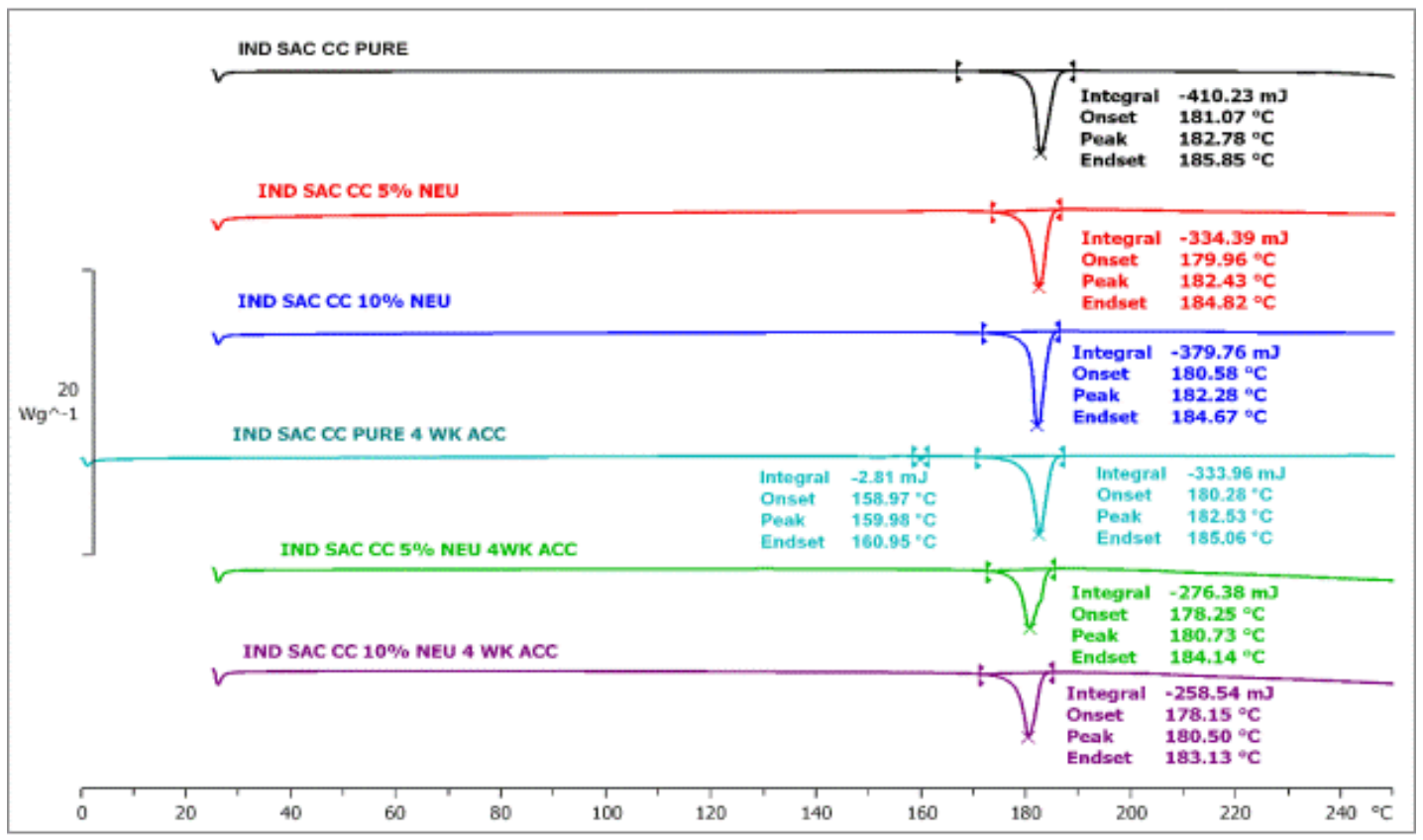

Figure 2a: DSC thermal profiles for: unmodified IND/SAC cocrystal, 5\% NEU blended cocrystal, 10\% NEU blended cocrystal (blue), unmodified cocrystal after stability testing , $5 \%$ NEU cocrystal after stability testing, 10\% NEU cocrystal after stability testing.

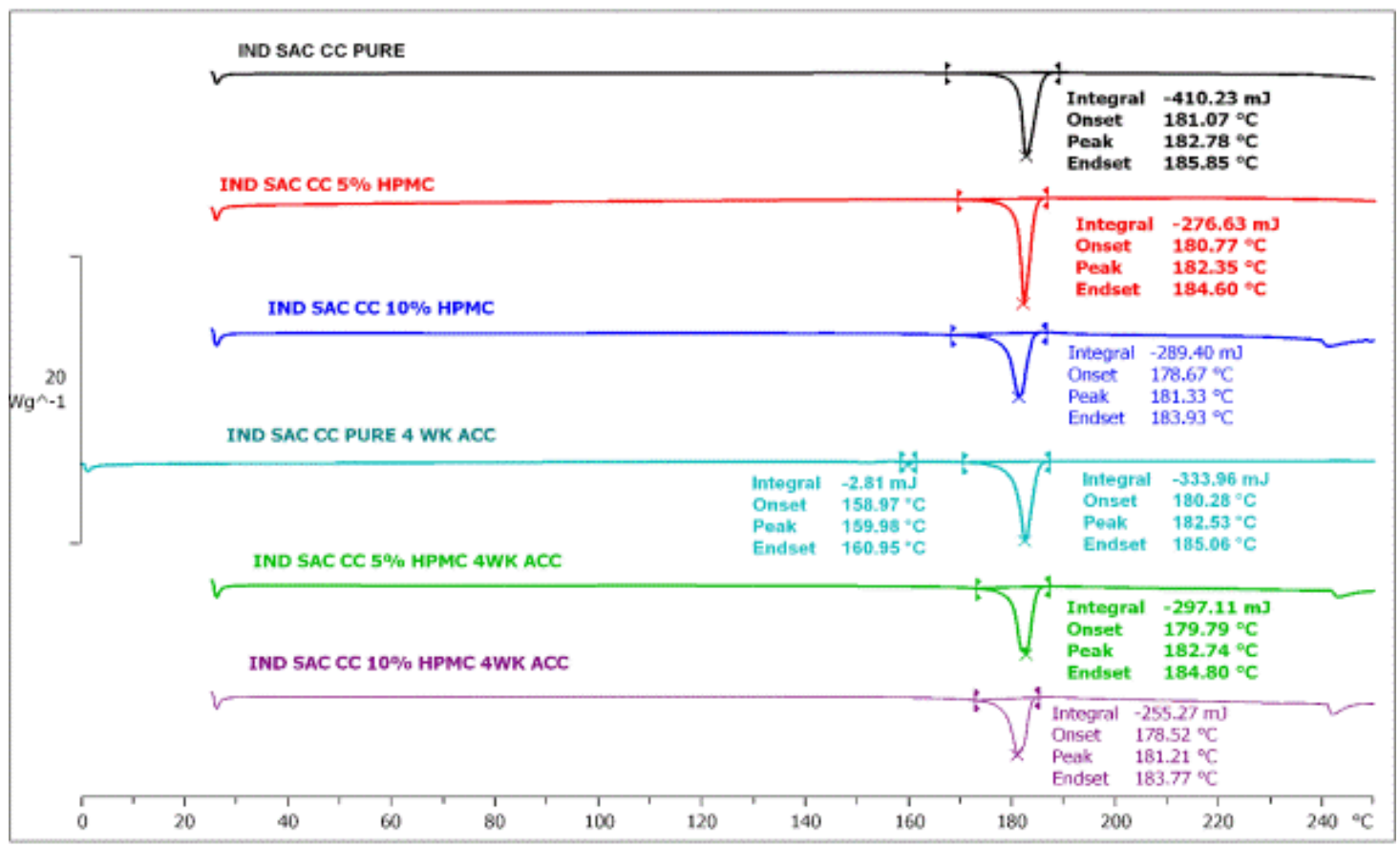


Figure 2b: DSC thermal profiles for: unmodified IND/SAC cocrystal, 5\% HPMC blended cocrystal, 10\% HPMC blended cocrystal, unmodified IND/SAC cocrystal after stability testing, 5\% HPMC cocrystal after stability testing, 10\% HPMC cocrystal after stability testing.

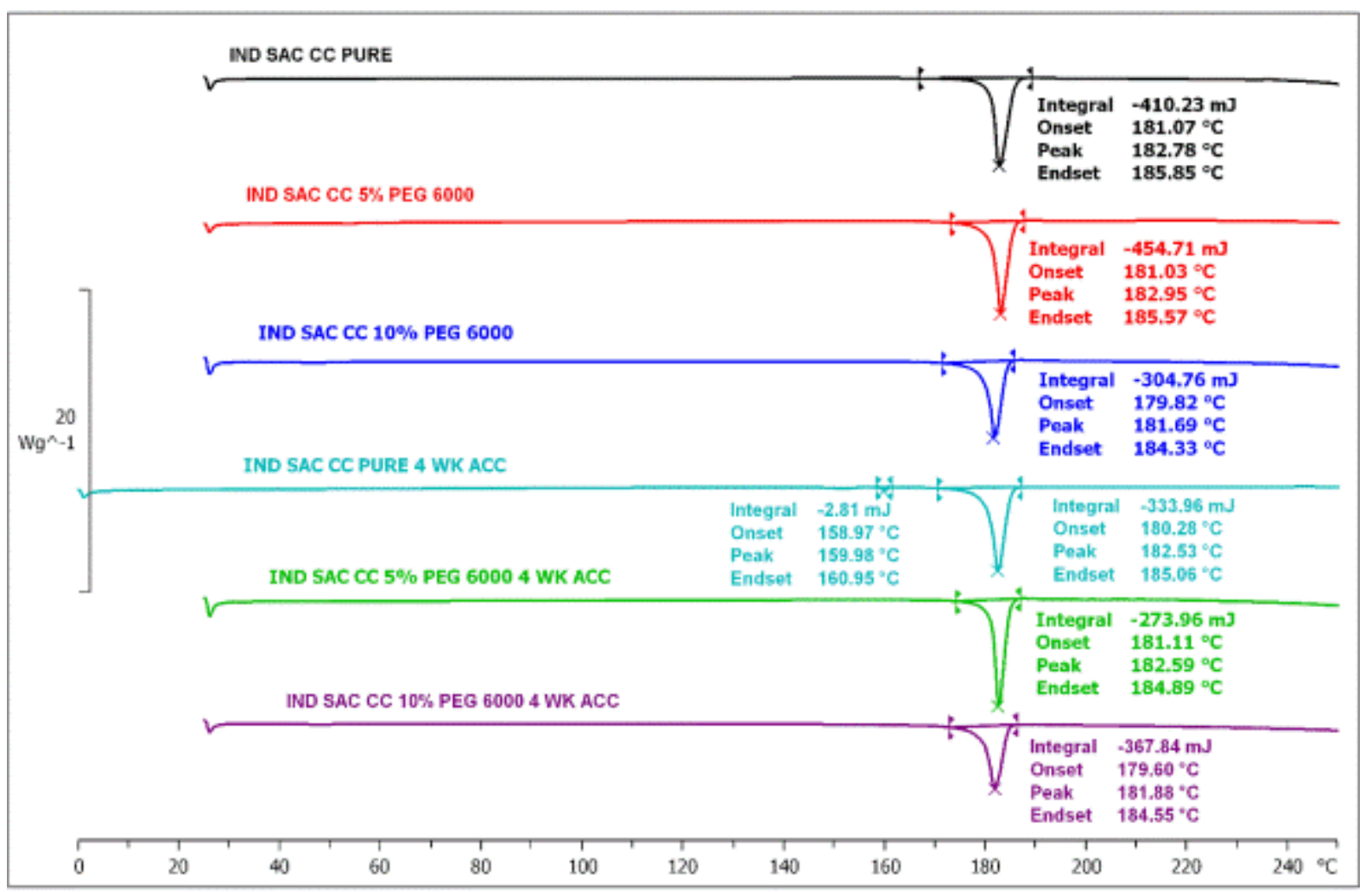

Figure 2c: DSC thermal profiles for: unmodified IND/SAC cocrystal (black), 5\% PEG blended cocrystal (red), 10\% PEG blended cocrystal (blue), unmodified IND/SAC cocrystal after stability testing (Teal), 5\% PEG cocrystal after stability testing (Green), 10\% PEG cocrystal after stability testing (violet).

\section{X-ray Analysis}

The synthesis and quality of the IND/SAC cocrystals were further evaluated through XRPD to identify the diffraction patterns of the bulk materials, physical mixture, unmodified IND/SAC 
cocrystals and excipient blended IND/SAC cocrystals. The analysis of bulk IND showed characteristic intensity peaks at $10.25,11.67,16.77,17.02,19.68,21.87,23.99,26.61^{\circ} 2 \theta$, while Bulk SAC displayed intensity peaks at $9.56,15.91,16.02,17.23,19.13,25.14^{\circ} 2 \theta$ (available in supporting information Figure S6). The diffractogram for the unmodified IND/SAC cocrystals were compared to previously reported structural data published in the CSD (REFCODE: UFERED). ${ }^{27}$ Our cocrystals were found to be identical to the CSD standard displaying characteristic intensity peaks at $5.43,10.89,14.42,21.22,25.45$, and $27.07^{\circ} 2 \theta$, with the absence of any peaks for the unreacted bulk components, indicating a high quality cocrystal (Figure 3).

However, as can be seen in Figure 4 after four weeks under accelerated conditions $\left(40 \pm 1{ }^{\circ} \mathrm{C}\right.$ and $75 \pm 1.5 \% \mathrm{RH})$ the IND/SAC cocrystal displayed newly formed peaks which did not match with that of the pure cocrystal. The newly formed peaks at $10.21,11.67,21.87^{\circ} 2 \theta$ correlate to peaks of IND, indicating the presence of recrystallized bulk IND. ${ }^{27}$ This further reinforces the idea that the cocrystal has begun to disassociate due to coformer dissolution, leaving excess IND in the sample.

The diffractograms for all excipient blended IND/SAC cocrystals, after being held for stability testing for four weeks can be seen in Figure 4 alongside that of the unmodified IND/SAC cocrystal before and after stability testing and bulk IND. As can be observed, all excipient blended cocrystals display identical structural data to that the pure cocrystal, indicating the extrusion process was a success and that the excipients have not interacted with the cocrystal. The absence of any peak for bulk IND suggests the excipient matrices have sufficiently protected the cocrystals from disassociation under elevated temperatures and humidity.

To further verify the percentage of the batch which successfully underwent cocrystallization Rietveld refinements were carried out for the pure and excipient blended cocrystals before and 
after stability testing. All samples were fitted to previously published structures for IND, SAC and IND/SAC cocrystal, obtained from the CSD. It is generally agreed that if the weighted profile $R$ factor $\left(\mathrm{R}_{\mathrm{wp}}\right)$ is within $3 \mathrm{x}$ the expected $R$ factor $\left(\mathrm{R}_{\mathrm{exp}}\right)$, the fitting can be considered accurate. ${ }^{40} \mathrm{In}$ this experiment, each Rietveld fitting is within this limit, with no fitting receiving a goodness of fit (GoF) above 3, so these results can be considered of excellent quality. It was found that both pure and excipient blended, IND/SAC cocrystals have a $100 \%$ match with the CSD reference structure. This supports the findings from the DSC and diffraction peak analysis and supports the hypothesis that feeding the added excipients into the extruder after cocrystallization has occurred restricts any possible interaction the excipient may have with the API-coformer pair, leaving the cocrystals chemical properties unaffected.

After being held under accelerated conditions for just 4 weeks, it was found that only $95.4 \%$ of the unmodified IND/SAC cocrystal was successfully fitted to the CSD reference structure. The excess $4.6 \%$ of the cocrystal was found to fit to the reference structure for pure IND, confirming that the cocrystal has partially dissociated leaving an amount of recrystallized IND. Except in one instance; all excipient blended cocrystals have maintained their crystallinity indicating that the excipient matrix has improved the physical stability for the IND/SAC cocrystal.

However, for the cocrystal blended with 10\% PEG 6000 the purity of the sample has decreased, as the fitting has dropped to $96.33 \%$. The unmatched peaks were found to fit to that of SAC, indicating that disassociation had taken place, but leaving excess SAC. This was not seen for the 5\% sample, suggesting the increase in PEG 6000 concentration may have led to a reaction with the cocrystal, causing chemical instability. This could be due to the fact that the melting point of PEG $6000\left(60-64^{\circ} \mathrm{C}\right)$, meaning it is far less thermally stable than the NEU and HPMC. Though PEG 6000 is generally regarded as stable in the crystalline form, it has been known to degrade at 
higher temperatures, facilitating its decomposition. ${ }^{41}$ It is plausible that, although the cocrystals were held at conditions lower than the melting point, the PEG 6000 started to soften under accelerated conditions, causing it to interact with the cocrystal constituents. Recent studies have shown that PEG has been known to react with IND in the molten state. Duong et al. (2015) demonstrated that each oxygen of the PEG molecule can form hydrogen bonds with two IND molecules. ${ }^{42}$ This is supported by a study from Chamsai et al. (2012) who found that interactions occurred between IND and the similar PEG 4000 under elevated conditions would lead to the formation of amorphous IND. ${ }^{43}$ This would explain why peaks for SAC were found as the formation of amorphous indomethacin would have left traces of unreacted SAC. The fact this was not seen in the $5 \%$ loaded IND/SAC cocrystals can be attributed to the lesser concentration added in the extrusion process, however, it is reasonable to believe that if left for longer, interactions between the molten PEG 6000 and IND is possible. Even though the excipient was added after the cocrystallization had taken place, the fact it is not thermally stable at higher temperatures and can form hydrogen bonds with IND means it has the potential to affect cocrystal purity.

The chemical instability witnessed with IND/SAC/PEG6000 cocrystals may be due to the PEG6000 being soluble with one of the bulk components. Phaechamud et al. (2010), noted solubilization of IND when using a 7:3 PEG4000:PEG400 mixture as an inert drug carrier and ElBadry and colleagues (2009), found that pure PEG 4000 also had a solubilizing effect when interacting with IND. ${ }^{44,45}$ Given these findings it is reasonable for one to assume that when left for a period of time, the IND began to solubilize within the PEG 6000 matrix causing the chemical instability noted in the XRPD analysis, which in turn led to an amount of recrystallized SAC being found in the sample after the 4-week period (All data for rietveld fittings displayed in supporting information Figures S8 and S9). 


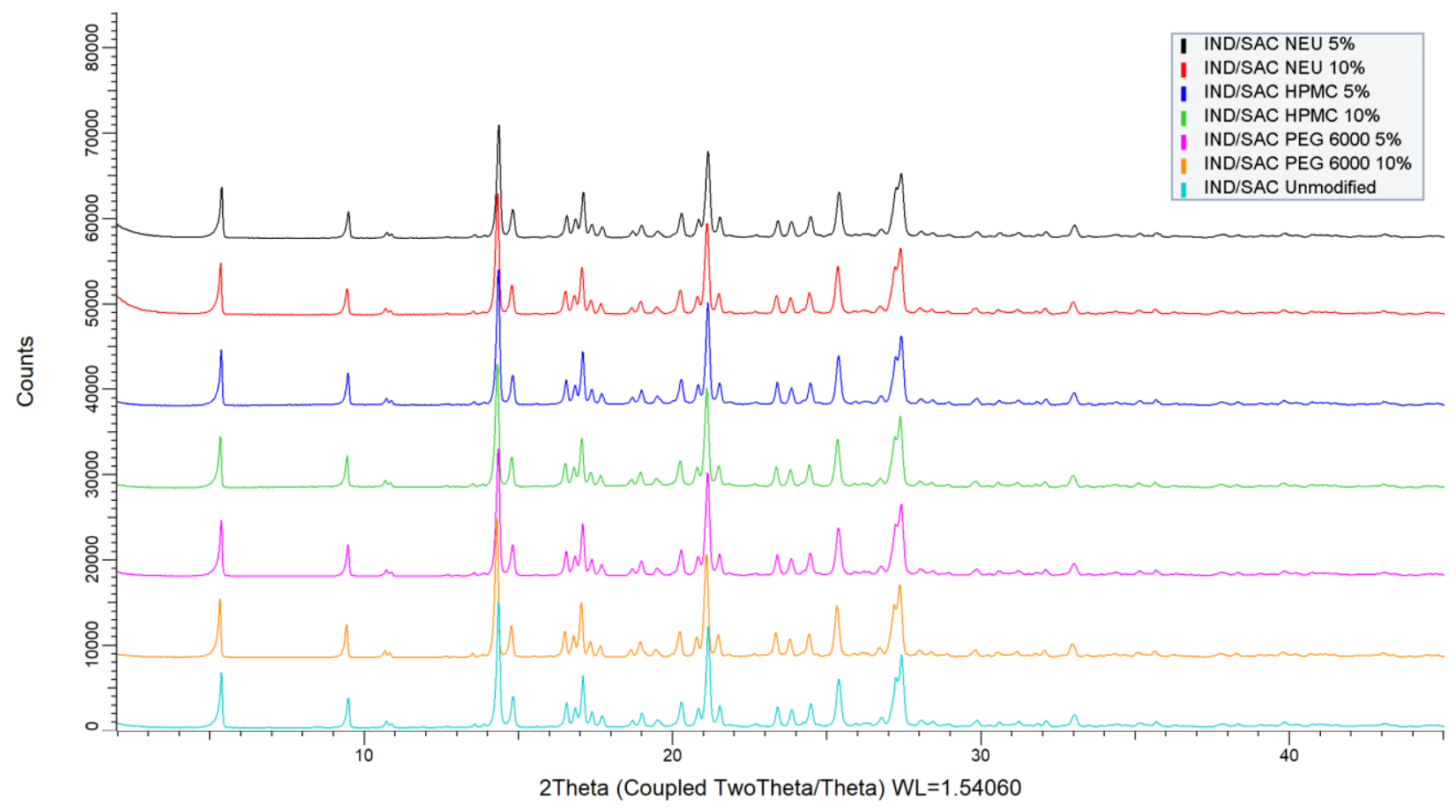

Figure 3. X-Ray diffractogram showing 5\% NEU blended, 10\% NEU blended, 5\% HPMC blended, 10\% HPMC blended, 5\% PEG 6000 blended, 10\% PEG 6000 blended and unmodified IND/SAC cocrystal after extrusion. 


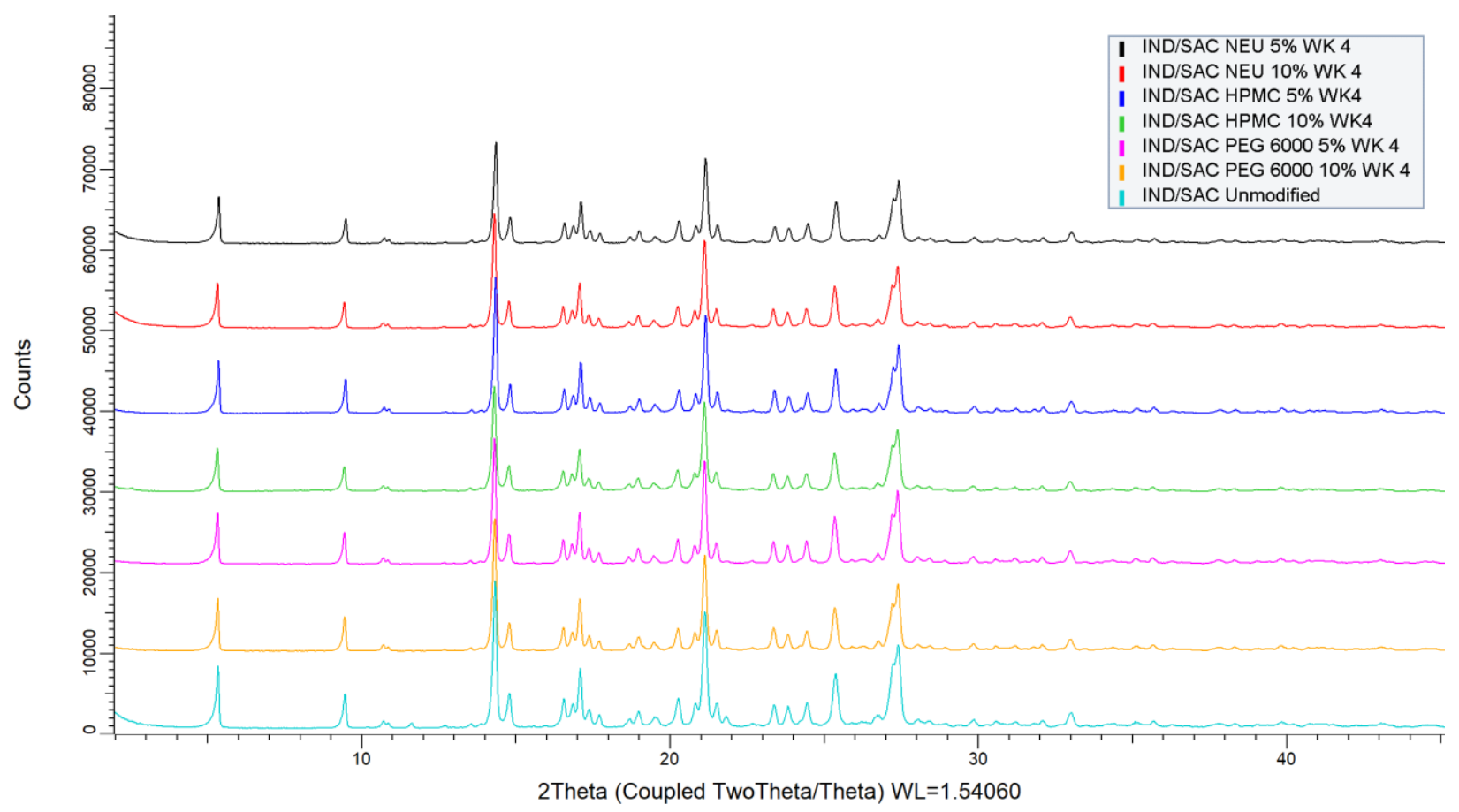

Figure 4. X-Ray diffractogram showing 5\% NEU blended, 10\% NEU blended, 5\% HPMC blended, 10\% HPMC blended, 5\% PEG 6000 blended, 10\% PEG 6000 blended and unmodified IND/SAC cocrystal after being held at accelerated conditions for 4 weeks.

\section{Particle size and morphology}

SEM analysis was employed to investigate the surface morphology of the bulk IND, physical mixtures and pure and excipient blended IND/SAC cocrystals. The micrograph of the bulk IND and unmodified IND/SAC cocrystals (Figures 5 and 6) show the bulk IND to be in the form of long irregularly shaped crystals, while the extruded IND/SAC cocrystals are shown to be equant shaped and in the form of agglomerates. However, a marked morphological difference can be seen between the unmodified IND/SAC cocrystal and those blended with NEU or HPMC excipients. While the unmodified IND/SAC cocrystals are in the form of freely moveable agglomerates, a dense matrix can be seen to have formed around the NEU and HPLC blended cocrystals. The 
excipient can be seen to work as a coating, forming a continuous layer around the cocrystals and causing them to amalgamate. The characteristic matrix is not present in the structure of the PEG 6000 blended cocrystals (Figures 5 and 6). This can be attributed to the fact that the PEG 6000 is crystalline, while NEU and HPMC are amorphous in structure. ${ }^{46}$ This could potentially explain why reduced crystallinity was observed in the PEG 6000 blended cocrystals after stability testing. In a similar study, where solid dispersions have been processed alongside polymers, it has been noted that the continuous layer can prevent the uptake of water molecules, thus dissuading hydrogen bonding. ${ }^{31,47}$ As both NEU and HPMC have excellent thermal stability there is little chance of this matrix breaking down under accelerated conditions, explaining why cocrystals blended with either excipients have showed excellent crystallinity after stability testing. This also supports XRPD data, as to why the 10\% PEG samples dissociated. The crystalline structure of PEG does not allow for the formation of such a layer, meaning moisture uptake and interaction with the API is more likely.

In the cases of the NEU and HPMC loaded cocrystals, the samples blended with $10 \%$ of excipients seem to exhibit a denser/more viscous matrix. This is confirmed through particle size analysis (Figures 7 and 8), where a greater percentage of $10 \%$ loaded cocrystals are greater than $250 \mu \mathrm{m}$. It should be noted that PEG 6000 blended samples have a far broader particle size distribution than the other samples. This may be due to the fact that the NEU and HPMC particles have amalgamated into a more organized matrix and that PEG 6000 has a greater particle size than the other excipients. 


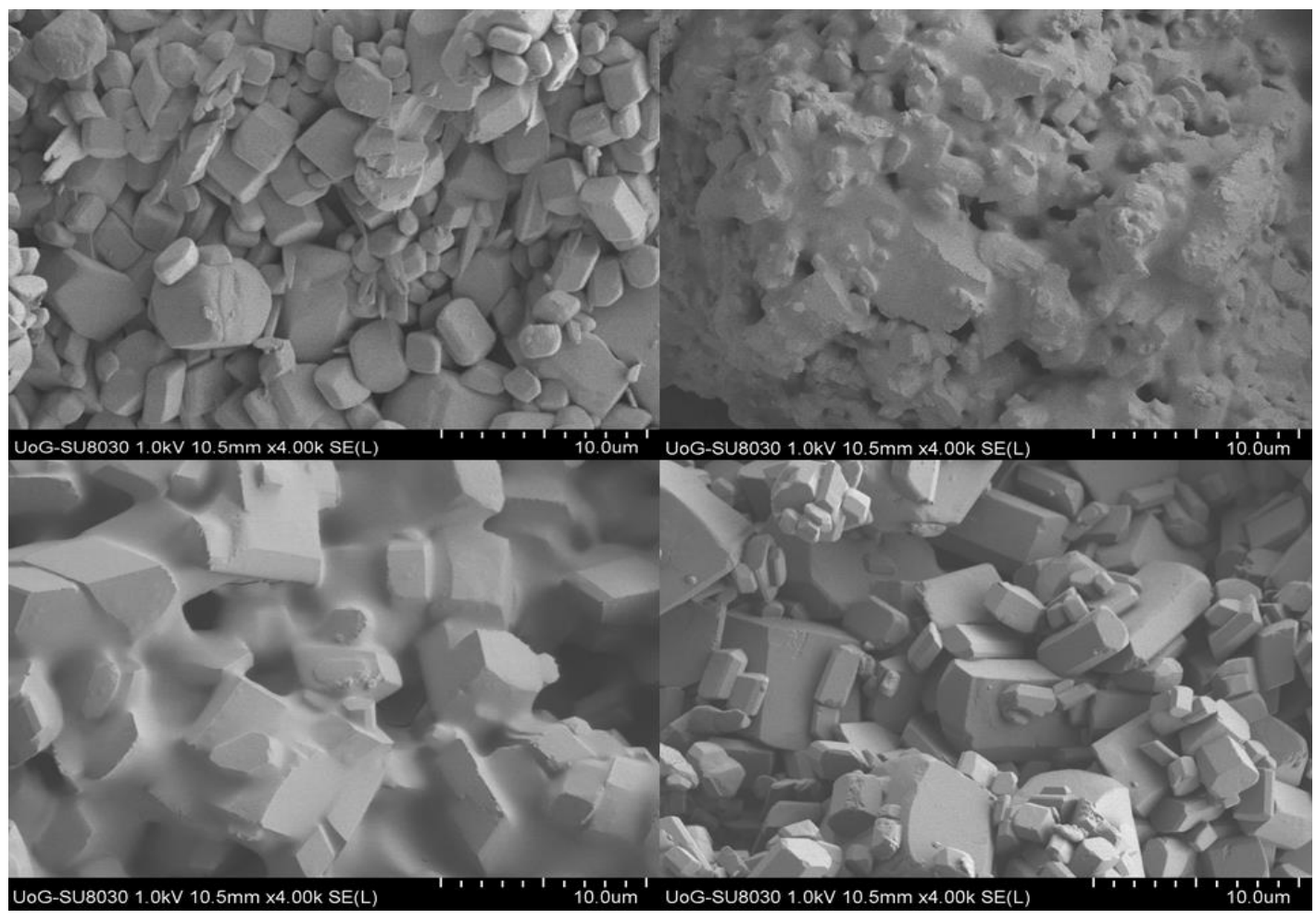

Figure 5. SEM images showing unmodified IND/SAC cocrystal (Top left), 5\% NEU blended (Top right), 5\% HPMC blended (Bottom left) and 5\% PEG 6000 Blended (Bottom right) IND/SAC cocrystals. 


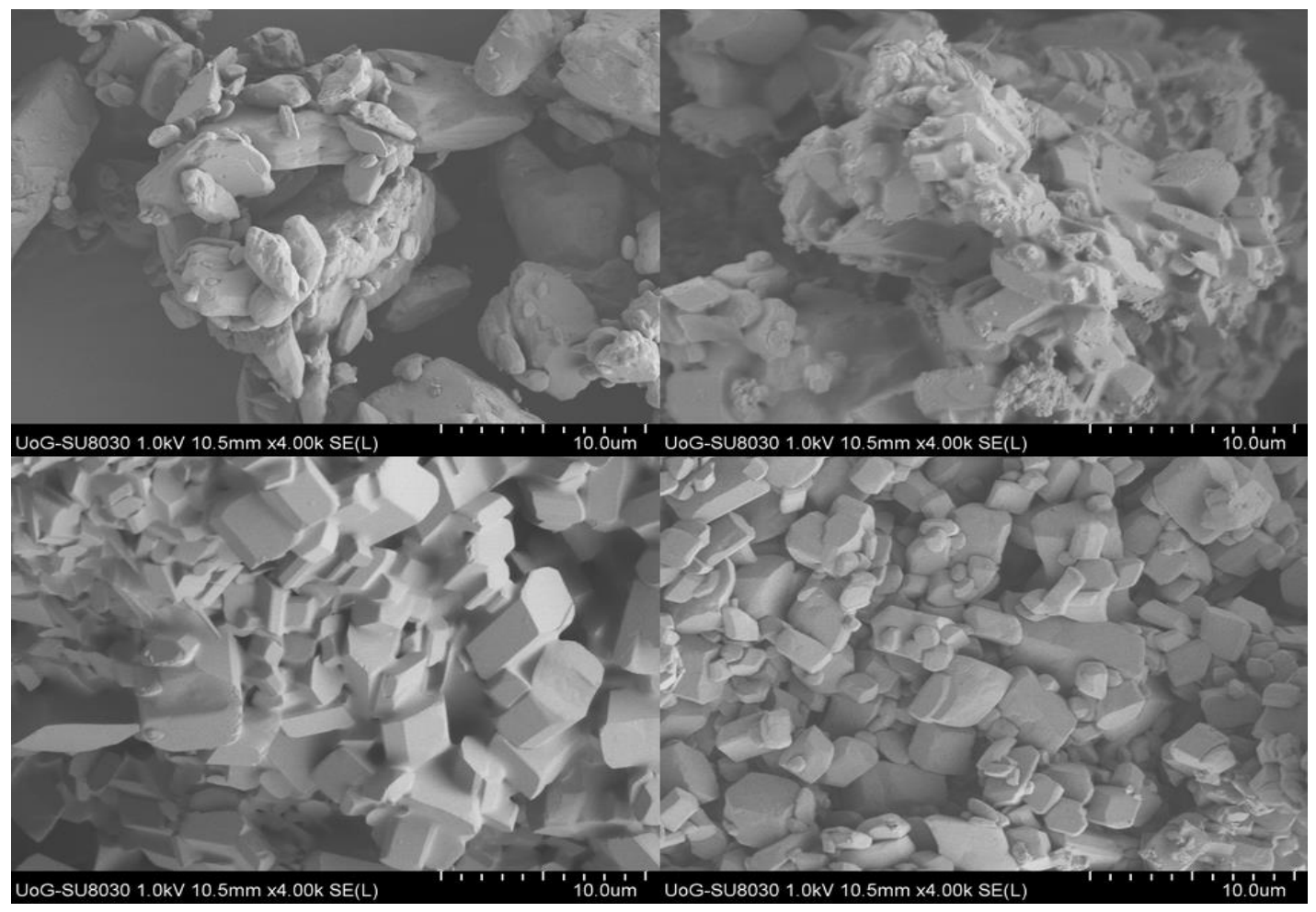

Figure 6. SEM images showing bulk IND (Top left), 10\% NEU blended (Top right), 10\% HPMC blended (Bottom left) and 10\% PEG 6000 Blended (Bottom right) IND/SAC cocrystals.

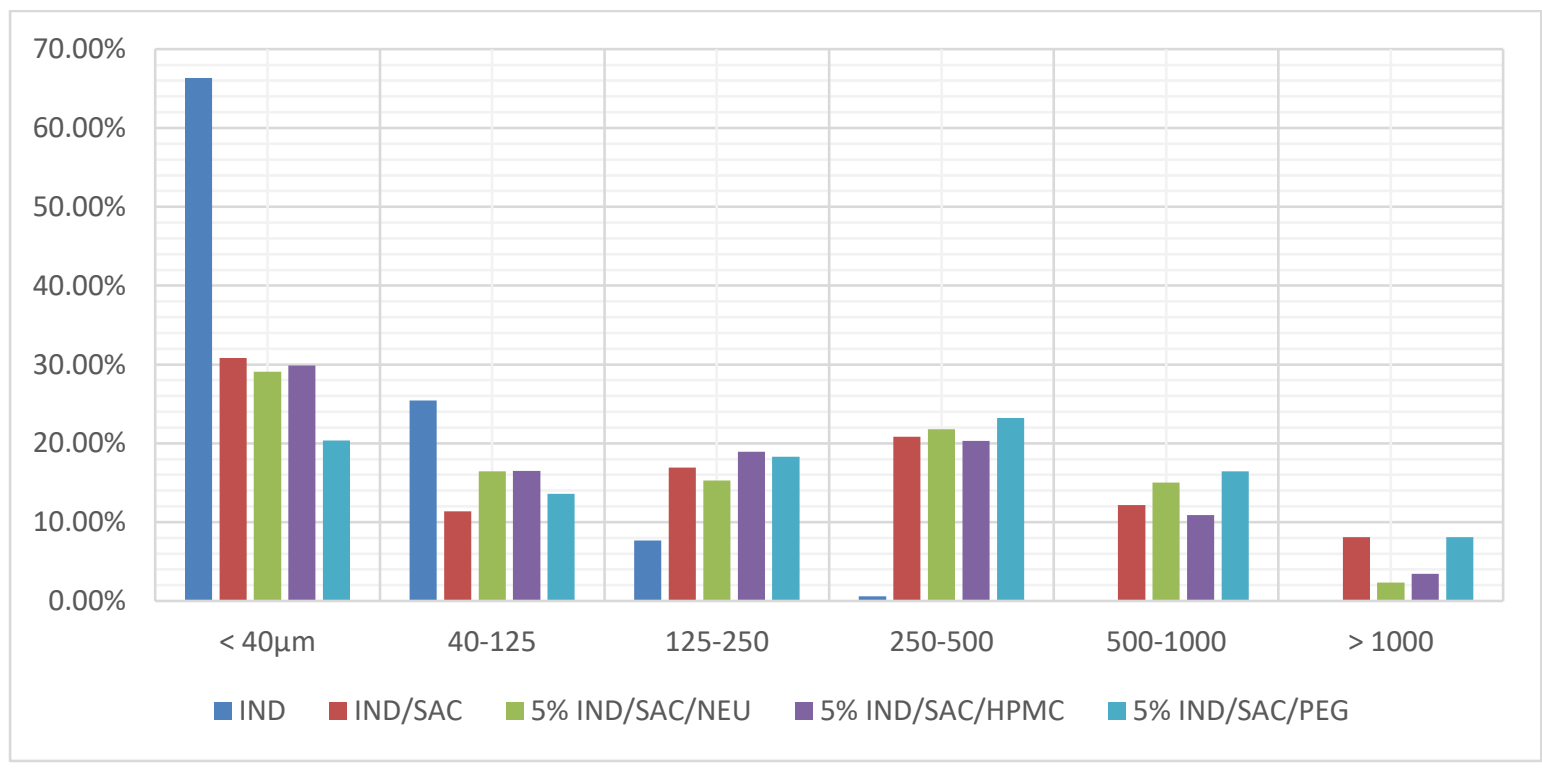

Figure 7. Particle size distributions for bulk IND, unmodified IND/SAC cocrystal and 5\% loaded excipient blended cocrystals. 


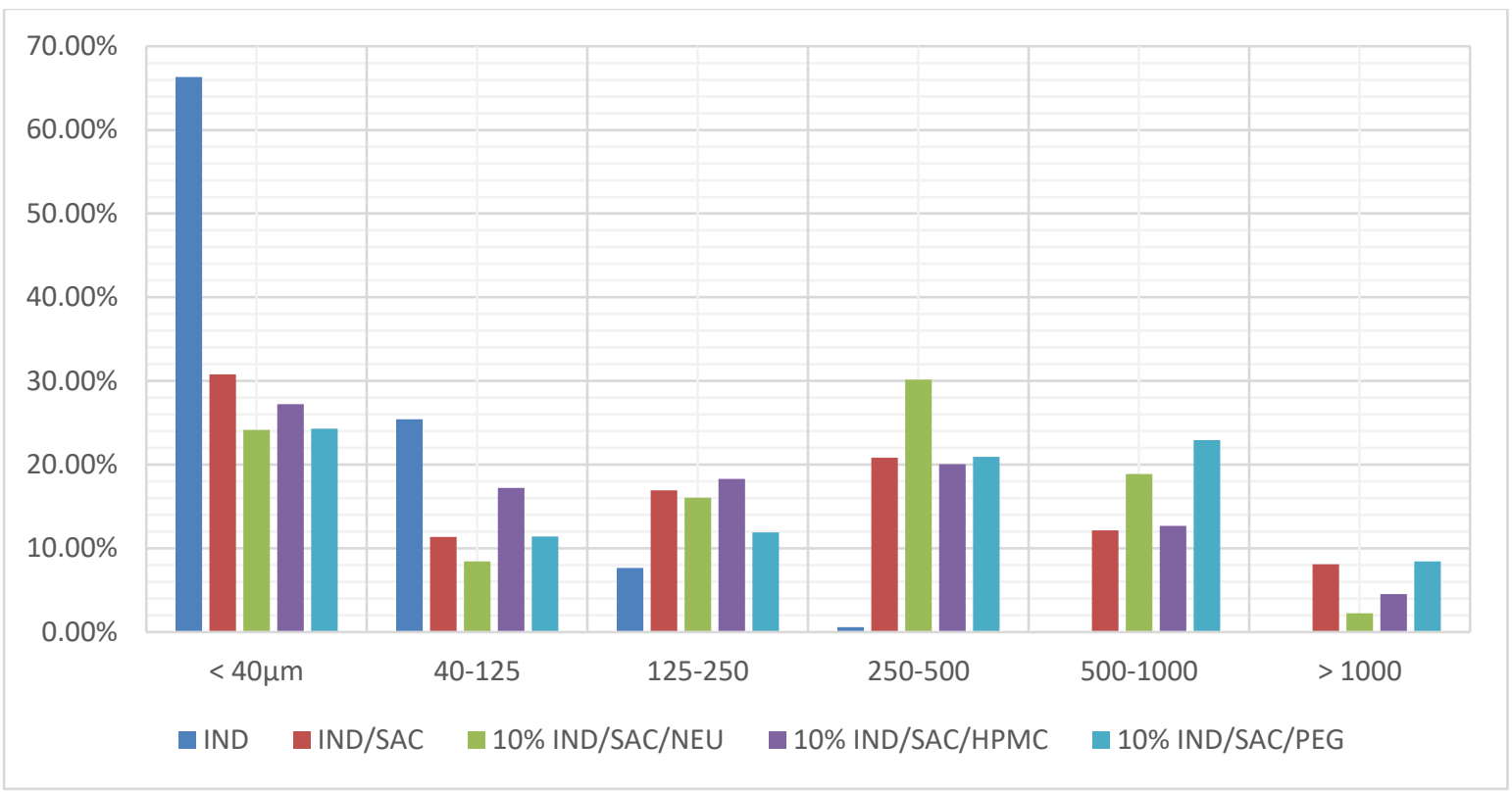

Figure 8. Particle size distributions for bulk IND, unmodified IND/SAC cocrystal and 10\% loaded excipient blended cocrystals.

\section{Dissolution studies}

As can be seen in Table 1, the unmodified cocrystal showed a moderate level of dissolution in the system displaying an IDR of $0.0562 \mathrm{mg} / \mathrm{min} / \mathrm{cm}^{2}$. Importantly, the pure co-crystal also showed a low deviation over the $n=3$ experiments of $4.75 \%$. The excipient coated cocrystals show similar dissolution rates to the unmodified IND/SAC cocrystal despite the additives, as shown in the dissolution profile comparison graph (Figure 9.). It can be noted from that the $10 \%$ HPMC cocrystal, and to a lesser degree the 5\% loaded cocrystal, has a much higher IDR value than the other excipients, indicating faster dissolution. From the images in Table 3 however, the $10 \%$ HPMC is showing the formation of a gel in the SDI. Typically, there will be a yellow or light blue runoff observed, if the sample was rapidly dissolving, but instead we can observe a swelling around a clear focal point (green dot within the surface). This confirms that the higher than expected result is due to the gelling of the HPMC. 
Gel was also seen in the flow cell post-dissolution. The formation of the gel leads to an inflated IDR as the gel traps the drug which then forms through the IDR zone. Hence, the inflated value of $0.1478 \mathrm{mg} / \mathrm{min} / \mathrm{cm}^{2}$ and the higher dissolution profile causing the high. The unpredictable gelling behaviour caused large variances in the 3 trials, causing the high standard deviation. The $5 \%$ HPMC coated cocrystals also displayed some gelling behaviour, however this occurred later in the dissolution than with the $10 \%$ coated crystals. This behaviour from HPMC samples has been noted before in SDI work and has been attributed to the higher viscosity of the polymer. ${ }^{48}$ This can be attributed to the lower concentration of HPMC present in the sample. Interestingly, the excipient coatings didn't appear to interfere with the dissolution of the co-crystal. This is likely a result of there being a low concentration of excipients in the mix, that each chosen excipient has excellent water solubility, and that as the excipient is only added to the extrusion process after the last kneading zone, the excipients are not interspersed throughout the crystal lattice and instead acts as - a protective matrix to the cocrystal. As the selected polymers are water soluble they do not negatively affect the dissolution of the cocrystal.

The in-vitro dissolution rate of the cocrystals co-processed with excipients was assessed, to ensure they were not hindering IND release. Here, USP-2 apparatus was utilized to evaluate the IND release rates of all excipient blended cocrystals. The excipient blended cocrystal samples were dissolved in a buffer solution alongside bulk IND and analysed the concentrations of each to find how easily the substance dissolves. IND is a BCS class II drug, meaning it has poor solubility as can be seen from (Figure 10.), where only 49\% of IND was dissolved in the medium after 2 hours. Cocrystallization of indomethacin has been reported to greatly enhances its dissolution rate, so it was no surprise that over $92.1 \%$ of IND dissolved after 2 hours in the cocrystal samples. ${ }^{49}$ The dissolution rate of the excipient blended samples closely matches that of the unmodified IND/SAC 
samples, with only the cocrystals blended with HPMC falling slightly below. The $10 \%$ HPMC loaded sample released $86.9 \%$ after a 2 -hour period, which is possibly the result of in-situ gelling as was seen in the SDI UV images. Gelling behaviour can slow drug dissolution as the gel will form on the sample surface and hinder penetration by the dissolution medium. The $5 \%$ HPMC loaded samples dissolution profile closely followed that of the unmodified IND/SAC cocrystals, which can be attributed to the lower concentrations. Pajander et al. (2012) found through UV SDI imaging that the higher viscosity grade, more rapidly the HPMC will swell, which leads to a thicker gel layer, which is more resistant toward the shear forces induced by the flow of the dissolution medium. ${ }^{50}$ Thus, the dissolution on HPMC blended IND/SAC cocrystals could potentially be quickened by using a lower viscosity HPMC. All other samples maintained a good dissolution rate, showing that the excipient matrix around the cocrystals will readily dissolve, thus not hindering the cocrystals dissolution rate. 
Table 1. Table showing the average intrinsic dissolution rate, standard deviations and UV images of IND/SAC cocrystal surface dissolution after 1, 10 and 20 minutes.

\begin{tabular}{|c|c|c|c|c|c|c|}
\hline Batch & 1 minute & 10 minutes & 20 minutes & Avg. IDR (mg/min/cm2) & St.Dev & \% St.Dev \\
\hline $\begin{array}{l}\text { Unmodified Co- } \\
\text { Crystal }\end{array}$ & & & & 0.0562 & 0.0027 & 4.75 \\
\hline $5 \%$ Neusillin & & & & 0.0569 & 0.0112 & 19.62 \\
\hline 5\% PEG 6000 & & & & 0.0706 & 0.0232 & 32.85 \\
\hline $5 \% \mathrm{HPMC}$ & & & & 0.0808 & 0.0275 & 34.07 \\
\hline 10\% Neusillin & & & & 0.0495 & 0.0114 & 23.11 \\
\hline $10 \%$ HPMC & & & & 0.1478 & 0.0461 & 31.16 \\
\hline 10\% PEG 6000 & & & & 0.0624 & 0.0131 & 21.02 \\
\hline Pure Indometh & & & & 0.0173 & 0.0017 & 0.10 \\
\hline
\end{tabular}




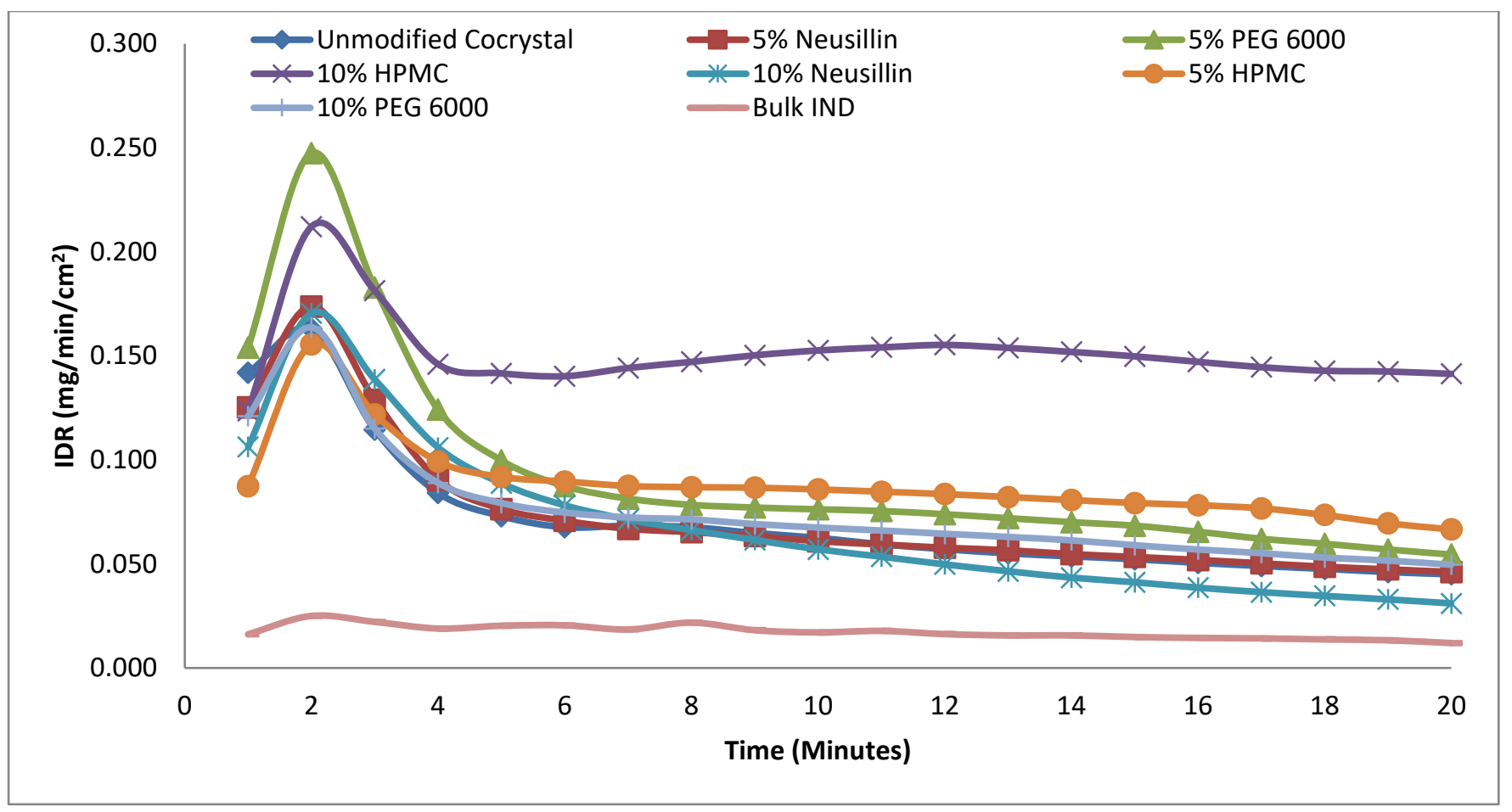

Figure 9. Intrinsic dissolution profiles of the pure and all excipient blended IND/SAC cocrystals.

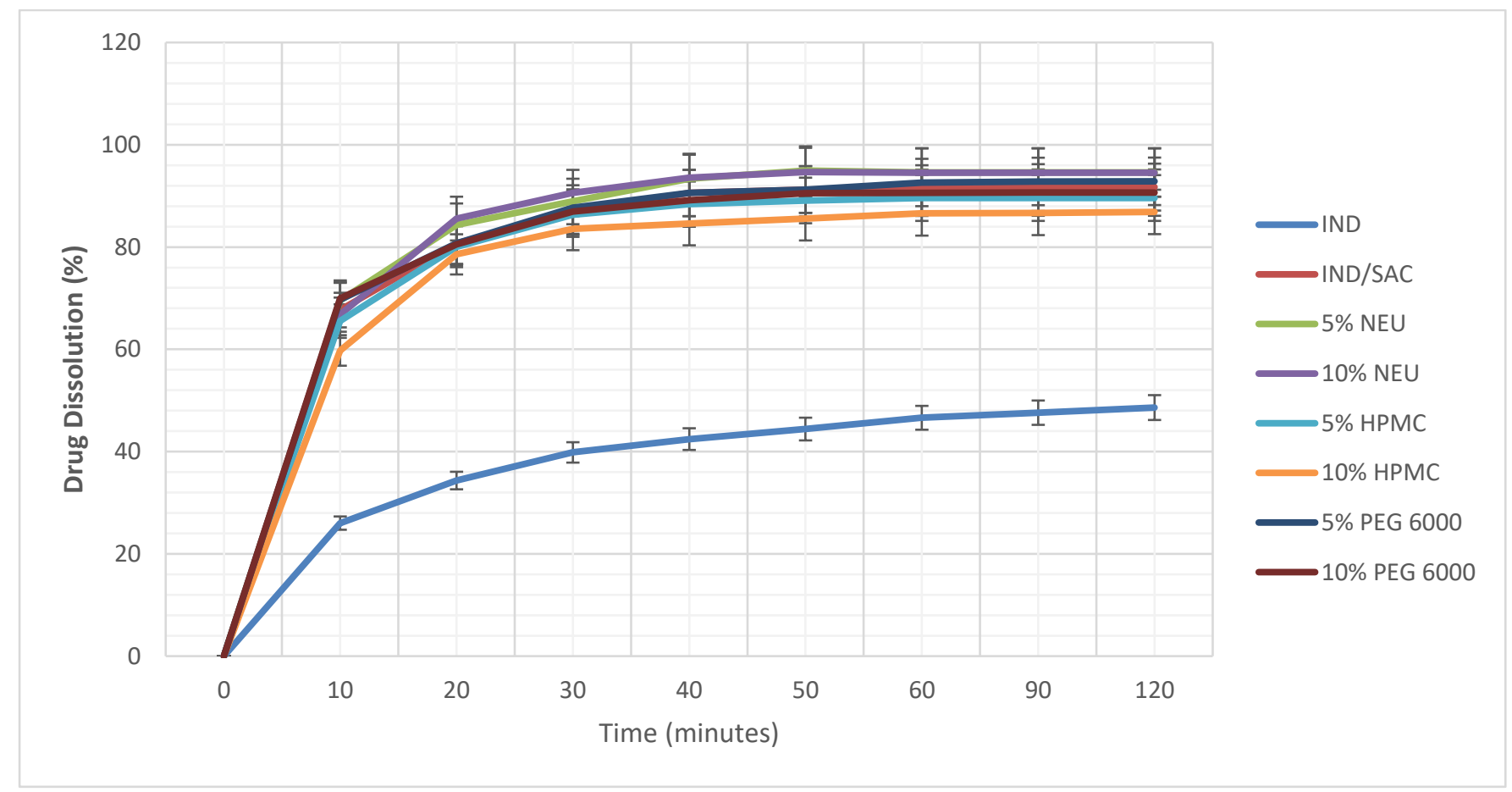

Figure 10. Cumulative release profiles of the pure and all excipient blended IND/SAC cocrystals. 


\section{Conclusion}

In conclusion, it has been successfully shown that co-processing an API/coformer blend in combination with a specific excipient can enhance the physicochemical stability of the resulting cocrystal. This study has demonstrated that blending the cocrystal with an NEU or HPMC allows the formation of a dense matrix, of which the cocrystal particles are embedded. It can be suggested that amorphous excipients are a superior option to crystalline excipients, which did not display the same protective matrix seen with the NEU and HPMC blended cocrystals. Furthermore, NEU and HPMC possess superior thermal stability than PEG 6000 and are not soluble with either cocrystal component, limiting interaction and preventing premature dissociation of the cocrystal. Excipients should be inert, non- miscible, have good thermal stability and a low viscosity. This study has demonstrated a successful mechanism for co-processing added excipients alongside the cocrystal by feeding them into the extruder conveying zones after cocrystallization has taken place, allowing for the cocrystal particles to become immersed in the liquefied excipient, before becoming embedded within them after solidification. 


\section{ASSOCIATED CONTENT}

\section{Supporting Information}

Images of extruder and Feeder set-up; Screw configuration, Table of process optimization trials, DSC thermograms of bulk IND, SCH, and their physical mixture; XRPD diffractograms of IND, $\mathrm{SCH}$, their physical mixtures and the physical mixtures of bulk constituents with the added excipients in 5\% and 10\% loadings; Table of rietveld fitting information's for unmodified and excipient loaded cocrystals before and after stability testing.

\section{AUTHOR INFORMATION}

\section{Corresponding Author}

*Tel.: +44(0)2083318440. Fax: +44(0)2083319805. E-mail: D.Douroumis@gre.ac.uk.

\section{Author Contributions}

The manuscript was written through contributions of all authors. All authors have given approval to the final version of the manuscript.

\section{Acknowledgments}

The authors would like to thank Pfizer Global Research \& Development for the support and teamwork 


\section{REFERENCES}

(1) Friščić, T.; Jones, W. Recent Advances In Understanding The Mechanism Of Cocrystal Formation Via Grinding. Cryst. Growth Des. 2009, 9, 1621-1637.

(2) Cherukuvada, S.; Kaur, R.; Guru Row, T. Co-Crystallization And Small Molecule Crystal Form Diversity: From Pharmaceutical To Materials Applications. CrystEngComm. 2016, 18, 8528-8555.

(3) Liu, Y.; Li, S.; Xu, J.; Zhang, H.; Guan, Y.; Jiang, H.; Huang, S.; Huang, H.; Wang, Z. Three Energetic 2,2',4,4',6,6'-Hexanitrostilbene Cocrystals Regularly Constructed By H-Bonding, ПStacking, And Van Der Waals Interactions. Cryst. Growth Des. 2018, 18, 1940-1943.

(4) Douroumis, D.; Ross, S.; Nokhodchi, A. Advanced Methodologies For Cocrystal Synthesis. Adv. Drug Deliv. Rev. 2017, 117, 178-195.

(5) Duggirala, N.; Perry, M.; Almarsson, Ö.; Zaworotko, M. Pharmaceutical Cocrystals: Along The Path To Improved Medicines. Chem. Commun. 2016, 52, 640-655.

(6) Gadade, D.; Pekamwar, S. Pharmaceutical Cocrystals: Regulatory And Strategic Aspects, Design And Development. Adv Pharm Bull. 2016, 6, 479-494.

(7) Bruni, G.; Maietta, M.; Berbenni, V.; Mustarelli, P.; Ferrara, C.; Freccero, M.; Grande, V.; Maggi, L.; Milanese, C.; Girella, A.; Marini, A. Mechanochemical Synthesis Of Bumetanide-4Aminobenzoic Acid Molecular Cocrystals: A Facile And Green Approach To Drug Optimization. A. J. Phys. Chem. B 2014, 118, 9180-9190. 
(8) Yadav, A.; Shete, A.; Dabke, A.; Kulkarni, P.; Sakhare, S. Co-Crystals: A Novel Approach

To Modify Physicochemical Properties Of Active Pharmaceutical Ingredients. Indian J. Pharm. Sci. $2009,71,359$.

(9) Karki, S.; Friščić, T.; Jones, W.; Motherwell, W. Screening For Pharmaceutical Cocrystal Hydrates Via Neat And Liquid-Assisted Grinding. Mol. Pharm. 2007, 4, 347-354.

(10) Thakuria, R.; Delori, A.; Jones, W.; Lipert, M.; Roy, L.; Rodríguez-Hornedo, N. Pharmaceutical Cocrystals And Poorly Soluble Drugs. Int. J. Pharm. 2013, 453, 101-125.

(11) Kalepu, S.; Nekkanti, V. Insoluble Drug Delivery Strategies: Review Of Recent Advances And Business Prospects. Acta Pharm Sin B. 2015, 5, 442-453.

(12) Jayasankar, A.; Roy, L.; Rodríguez-Hornedo, N. Transformation Pathways Of Cocrystal Hydrates When Coformer Modulates Water Activity. J. Pharm. Sci. 2010, 99, 3977-3985.

(13) Izutsu, K.; Koide, T.; Takata, N.; Ikeda, Y.; Ono, M.; Inoue, M.; Fukami, T.; Yonemochi, E. Characterization And Quality Control Of Pharmaceutical Cocrystals. Chem. Pharm. Bull. 2016, $64,1421-1430$.

(14) Eddleston, M.; Thakuria, R.; Aldous, B.; Jones, W. An Investigation Of The Causes Of Cocrystal Dissociation At High Humidity. J. Pharm. Sci. 2014, 103, 2859-2864.

(15) Bond, A.; Boese, R.; Desiraju, G. On The Polymorphism Of Aspirin: Crystalline Aspirin As Intergrowths Of Two "Polymorphic” Domains. Angew. Chem. Int. Ed. 2007, 46, 618-622.

(16) Liu, X.; Lu, M.; Guo, Z.; Huang, L.; Feng, X.; Wu, C. Improving The Chemical Stability Of Amorphous Solid Dispersion With Cocrystal Technique By Hot Melt Extrusion. Pharm. Res. 2011, 29, 806-817. 
(17) Boksa, K.; Otte, A.; Pinal, R. Matrix-Assisted Cocrystallization (MAC) Simultaneous Production And Formulation Of Pharmaceutical Cocrystals By Hot-Melt Extrusion. J. Pharm. Sci. 2014, 103, 2904-2910.

(18) Li, S.; Yu, T.; Tian, Y.; McCoy, C.; Jones, D.; Andrews, G. Mechanochemical Synthesis Of Pharmaceutical Cocrystal Suspensions Via Hot Melt Extrusion: Feasibility Studies And Physicochemical Characterization. Mol. Pharm. 2016, 13, 3054-3068.

(19) Hernández Espinell, J.; López-Mejías, V.; Stelzer, T. Revealing Polymorphic Phase Transformations In Polymer-Based Hot Melt Extrusion Processes. Cryst. Growth Des. 2018, 18, 1995-2002.

(20) Dhumal, R.; Kelly, A.; York, P.; Coates, P.; Paradkar, A. Cocrystalization And Simultaneous Agglomeration Using Hot Melt Extrusion. Pharm. Res. 2010, 27, 2725-2733.

(21) Korde, S.; Pagire, S.; Pan, H.; Seaton, C.; Kelly, A.; Chen, Y.; Wang, Q.; Coates, P.; Paradkar, A. Continuous Manufacturing Of Cocrystals Using Solid State Shear Milling Technology. Cryst. Growth Des. 2018, 18, 2297-2304.

(22) Allgeier, M.; Piper, J.; Hinds, J.; Yates, M.; Kolodsick, K.; Meury, R.; Shaw, B.; Kulkarni, M.; Remick, D. Isolation And Physical Property Optimization Of An Amorphous Drug Substance Utilizing A High Surface Area Magnesium Aluminometasilicate (Neusilin ${ }^{\circledR}$ US2). J. Pharm. Sci. 2016, 105, 3105-3114.

(23) Gupta, M.; Vanwert, A.; Bogner, R. Formation Of Physically Stable Amorphous Drugs By Milling With Neusilin. J. Pharm. Sci. 2003, 92, 536-551. 
(24) Groom, C.; Bruno, J.; Lightfoot, M.; Ward, S. The Cambridge Structural Database. Acta Crystallogr., Sect. B: Struct. Sci 2016, 72, 171-179.

(25) Chen, X.; Morris, K.; Griesser, U.; Byrn, S.; Stowell, J. Reactivity Differences Of Indomethacin Solid Forms With Ammonia Gas. J. Am. Chem. Soc. 2002, 124, 15012-15019.

(26) Wardell, J.; Low, J.; Glidewell, C. Saccharin, Redetermined At $120 \mathrm{~K}$ : A ThreeDimensional Hydrogen-Bonded Framework. Acta Crystallogr. Sect. E: Struct. Rep. Online 2005, 61, 1944-o1946.

(27) Basavoju, S.; Boström, D.; Velaga, S. Indomethacin-Saccharin Cocrystal: Design, Synthesis And Preliminary Pharmaceutical Characterization. Pharm. Res. 2007, 25, 530-541.

(28) ICH. Q1A(R2). Stability testing of new drug substances and products (2nd revision). ICH Steering Committee; 2003.

(29) Moradiya, H.; Islam, M.; Scoutaris, N.; Halsey, S.; Chowdhry, B.; Douroumis, D. Continuous Manufacturing Of High Quality Pharmaceutical Cocrystals Integrated With Process Analytical Tools For In-Line Process Control. Cryst. Growth Des. 2016, 16, 3425-3434.

(30) Dhumal, R.; Kelly, A.; York, P.; Coates, P.; Paradkar, A. Cocrystalization And Simultaneous Agglomeration Using Hot Melt Extrusion. Pharm. Res. 2010, 27, 2725-2733.

(31) Capece, M.; Davé, R. Enhanced Physical Stability Of Amorphous Drug Formulations Via Dry Polymer Coating. J. Pharm. Sci. 2015, 104, 2076-2084.

(32) Qiu, S.; Lai, J.; Guo, M.; Wang, K.; Lai, X.; Desai, U.; Juma, N.; Li, M. Role Of Polymers In Solution And Tablet-Based Carbamazepine Cocrystal Formulations. CrystEngComm 2016, 18 , 2664-2678. 
(33) Ross, S.; Lamprou, D.; Douroumis, D. Engineering And Manufacturing Of Pharmaceutical Co-Crystals: A Review Of Solvent-Free Manufacturing Technologies. Chem. Commun. 2016, 52, $8772-8786$.

(34) Ross, S.; Malamatari, M.; Douroumis, D.; Velaga, S. Experimental Cocrystal Screening and Solution Based Scale-Up Cocrystallization Methods. Advanced Drug Delivery Reviews 2017, $117,162-177$.

(35) Ziegler, G.; Aguilar, C. Residence Time Distribution In A Co-Rotating, Twin-Screw Continuous Mixer By The Step Change Method. J. Food Eng. 2003, 59, 161-167.

(36) Shewale, S.; Shete, A.; Doijad, R.; Kadam, S.; Patil, V.; Yadav, A. Formulation And Solid State Characterization Of Nicotinamide-Based Co-Crystals Of Fenofibrate. Indian J. Pharm. Sci. $2015,77,328$.

(37) Eddleston, M.; Madusanka, N.; Jones, W. Cocrystal Dissociation In The Presence Of Water: A General Approach For Identifying Stable Cocrystal Forms. J. Pharm. Sci. 2014, 103, 28652870 .

(38) Khankari, R.; Law, D.; Grant, D. Determination Of Water Content In Pharmaceutical Hydrates By Differential Scanning Calorimetry. Int. J. Pharm. 1992, 82, 117-127.

(39) McPhillips, H.; Craig, D.; Royall, P.; Hill, V. Characterisation Of The Glass Transition Of HPMC Using Modulated Temperature Differential Scanning Calorimetry. Int. J. Pharm. 1999, $180,83-90$.

(40) Toby, B. R Factors In Rietveld Analysis: How Good Is Good Enough?. Powder Diffr. 2006, 21, 67-70. 
(41) Otal, E.; Mantzavinos, D.; Delgado, M.; Hellenbrand, R.; Lebrato, J.; Metcalfe, I.; Livingston, A. Integrated Wet Air Oxidation And Biological Treatment Of Polyethylene GlycolContaining Wastewaters. J. Chem. Technol. Biotechnol. 1997, 70, 147-156.

(42) Duong, T.; Van Humbeeck, J.; Van den Mooter, G. Crystallization Kinetics Of Indomethacin/Polyethylene Glycol Dispersions Containing High Drug Loadings. Mol. Pharm. $2015,12,2493-2504$.

(43) Chamsai, B.; Sriamornsak, P. Physical Stabilizing Effect Of Biopolymers On Solid Dispersions Containing Indomethacin And Polyethylene Glycol. Adv. Mater. Res. 2012, 506, $307-$ 310.

(44) Phaechamud, T.; Mesnukul, A.; Yodkhum, K.; Mahadlek, J. Characterization Of Indomethacin Release From Polyethylene Glycol Tablet Fabricated With Mold Technique. Indian J. Pharm. Sci. 2010, 72, 92.

(45) El-Badry, M.; Fetih, G.; Fathy, M. Improvement Of Solubility And Dissolution Rate Of Indomethacin By Solid Dispersions In Gelucire 50/13 And PEG4000. Saudi Pharm J.2009, 17, 217-225.

(46) Klumperman, B. Aqueous Polymeric Coatings For Pharmaceutical Dosage Forms (Drugs And The Pharmaceutical Sciences). Macromol. Chem. Phys. 2009, 210, 299-299.

(47) Hoashi, Y.; Tozuka, Y.; Takeuchi, H. Solventless Dry Powder Coating For Sustained Drug Release Using Mechanochemical Treatment Based On The Tri-Component System Of Acetaminophen, Carnauba Wax And Glidant. Drug Dev. Ind. Pharm. 2012, 39, 259-265. 
(48) Østergaard, J.; Lenke, J.; Sun, Y.; Ye, F. UV Imaging For In Vitro Dissolution And Release Studies: Intial Experiences. Dissolut Technol. 2014, 21.

(49) Alhalaweh, A.; Sokolowski, A.; Rodríguez-Hornedo, N.; Velaga, S. Solubility Behavior And Solution Chemistry Of Indomethacin Cocrystals In Organic Solvents. Cryst. Growth Des. $2011,11,3923-3929$.

(50) Pajander, J.; Baldursdottir, S.; Rantanen, J.; Østergaard, J. Behaviour Of HPMC Compacts Investigated Using UV-Imaging. Int. J. Pharm. 2012, 427, 345-353. 


\section{For Table of Contents Use Only}

Co - processing of pharmaceutical cocrystals for high quality and enhanced physicochemical stability

Steven A. Ross ${ }^{1}$, Adam Ward ${ }^{2}$, Pat Basford ${ }^{3}$, Mark McAllister ${ }^{3}$, Dennis Douroumis ${ }^{1,4 \dagger}$

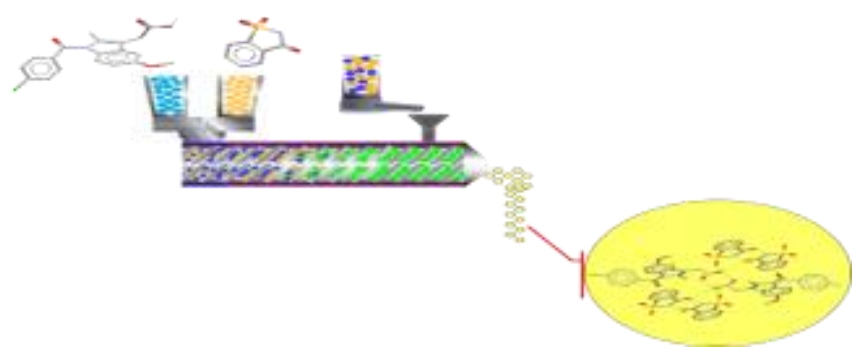

\section{Synopsis}

Physical and chemical stability of indomethacin-saccharin (IND-SAC) cocrystals synthesised via hot-melt extrusion (HME) was enhanced through co - processing with inert excipients at the final kneading zone. Characterization revealed the added excipients did not interact with the cocrystals and instead embedder them in a vicious, protective matrix. Cocrystals co-processed alongside Neusilin and hydroxypropyl methylcellulose (HPMC) displayed superior stability under accelerated conditions.

$\dagger$ Correspondence: Medway School of Science, Universities of Greenwich \& Kent, Medway Campus, Chatham Maritime, Kent ME4 4TB, UK. Tel.: +44 (0) 208331 8440; Fax: +44 (0) 208 331 9805.E-mail address: D.Douroumis@gre.ac.uk 